\title{
A Multi-scale Model of Dislocation Plasticity in $\alpha$-Fe: Incorporating Temperature, Strain Rate and Non-Schmid Effects
}

\author{
H. Lim ${ }^{\mathrm{a}, *}$, L. M. Hale ${ }^{\mathrm{b}}$, J. A. Zimmerman ${ }^{\mathrm{c}}$, C. C. Battaile ${ }^{\mathrm{a}}$, C. R. Weinberger ${ }^{\mathrm{d}}$ \\ ${ }^{a}$ Department of Computational Materials and Data Science, Sandia National Laboratories, Albuquerque, NM, 87185 \\ ${ }^{b}$ Thermodynamics and Kinetics Group, National Institute of Standards and Technology, Gaithersburg, MD, 20899 \\ ${ }^{c}$ Department of Mechanics of Materials, Sandia National Laboratories, Livermore, CA, 94551 \\ ${ }^{d}$ Department of Mechanical Engineering and Mechanics, Drexel University, Philadelphia, PA, 19104
}

\begin{abstract}
In this work, we develop an atomistically informed crystal plasticity finite element (CP-FE) model for body-centered-cubic (BCC) $\alpha$-Fe that incorporates non-Schmid stress dependent slip with temperature and strain rate effects. Based on recent insights obtained from atomistic simulations, we propose a new constitutive model that combines a generalized non-Schmid yield law with aspects from a line tension (LT) model for describing activation enthalpy required for the motion of dislocation kinks. Atomistic calculations are conducted to quantify the non-Schmid effects while both experimental data and atomistic simulations are used to assess the temperature and strain rate effects. The parameterized constitutive equation is implemented into a BCC CP-FE model to simulate plastic deformation of single and polycrystalline Fe which is compared with experimental data from the literature. This direct comparison demonstrates that the atomistically informed model accurately captures the effects of crystal orientation, temperature and strain rate on the flow behavior of single crystal Fe. Furthermore, our proposed CP-FE model exhibits temperature and strain rate dependent flow and yield surfaces in polycrystalline Fe that deviate from conventional CP-FE models based on Schmid's law.
\end{abstract}

Keywords: Crystal plasticity, atomistics, constitutive behavior, finite elements

\section{Introduction}

Developing a constitutive material model that captures the underlying physics is important to accurately predict and design for structural applications. This has proven more challenging for

\footnotetext{
*Corresponding author

Email address: hnlim@sandia.gov (H. Lim)
} 
body-centered-cubic (BCC) metals, i.e. $\alpha$-Fe and refractory metals, mainly due to the complexity associated with the mechanisms for plastic flow in BCC metals. BCC metals exhibit distinctive mechanical characteristics that deviate from face-centered-cubic (FCC) metals, e.g. non-Schmid yield behavior, strong temperature and strain rate dependence and the ambiguity of the slip planes on which slip occurs (Christian, 1983; Seeger, 1981; Weinberger et al., 2013a). At low temperatures, the yield behavior of BCC metals is strongly dependent on the glide of a $/ 2\langle 111\rangle$ screw dislocations. The Peierls barrier associated with the glide of these screw dislocations is typically large enough that the dislocations do not freely move until subjected to a moderate temperature and/or stress. This barrier to dislocation motion directly results in flow being temperature and rate dependent and results in yield surfaces that violate Schmid's law.

Accurately capturing the nature of the dislocations in these materials requires an atomistic representation due to the narrow and non-planar core structure. The stable screw dislocation cores in BCC metals have long been predicted and calculated with atomistic methods to be nonplanar in nature, with the core equally spread out on three different $\{110\}$ crystallographic planes (Hirsch, 1960; Duesbery et al., 1973; Duesbery and Vitek, 1998). This gives the stable core a threefold rotational symmetry consistent with the symmetry associated with the $\langle 111\rangle$ crystallographic projection. Additionally, the spreading of the core allows it to be influenced and distorted by stresses both parallel and perpendicular to the slip direction (Duesbery and Vitek, 1998; Ito and Vitek, 2001; Gröger et al., 2008a). It is these properties of the dislocation core structure that lead to deviations from Schmid's law, i.e. the critical stress to move the dislocation is influenced by the entire stress state and not just the shear stress acting on the slip plane in the slip direction.

Investigations of the atomic structure of the core using classical potentials have revealed a few features associated with the core that are observed to be potential dependent and influence the resulting slip behavior. Depending on the potential, the stable core may or may not have a twofold inversion symmetry associated with the $\langle 110\rangle$ directions in addition to the three-fold rotational symmetry. Cores that have symmetry are referred to as compact cores and those that lack it are referred to as either degenerate or polarized cores. This lack of symmetry of the core structure allows for certain kinks associated with particular degenerate (polarized) core arrangements to be more favorable than others, which in turn can influence the observed slip direction (Duesbery et al., 1973; Rao and Woodward, 2001; Wang et al., 2002; Rodney, 2007). 
In addition to the non-planar stable core, a number of classical potentials predict a metastable planar core structure (Takeuchi and Kuramoto, 1975; Takeuchi, 1979; Gordon et al., 2010). This planar core, referred to as a split core, is associated with the dislocation being completely on one $\{110\}$ plane at a position halfway between two of the non-planar core position. The presence of this metastable core results in the Peierls barrier associated with $\{110\}$ planar motion of the dislocation having a double energy barrier as opposed to the more classic single peak barriers. Recent works have shown that the presence of the split core influences the slip nature of the dislocations (Gordon et al., 2010, 2011; Hale et al., 2014b). Density functional theory (DFT) studies show that for all of the BCC transition metals, the stable core is predicted to be compact, and the split core is predicted to be unstable (Ventelon and Willaime, 2007; Weinberger et al., 2013b). Because of this, we have searched for and found a classical potential for BCC Fe that captures the correct core stabilities. This was not a trivial pursuit, as other works have noted a tendency for BCC iron potentials that predict the compact core to also predict the split core (Gordon et al., 2010, 2011).

Turning to the continuum scale, various yield criteria have been proposed to capture nonSchmid effects in BCC (Gröger et al., 2008b; Yalcinkaya et al., 2008; Koester et al., 2012) and other metals (Qin and Bassani, 1992a,b; Dao and Asaro, 1993; Steinmann et al., 1998). This nonSchmid yield behavior has been incorporated into larger scale models for BCC metals using either the crystal plasticity-finite element (CP-FE) model (Weinberger et al., 2012; Lim et al., 2013, 2015), or discrete dislocation dynamics (Wang and Beyerlein, 2010; Srivastava et al., 2013). Another class of BCC models focuses on capturing the temperature and strain rate effects associated with the slip activation of the BCC dislocations (Kocks et al., 1975; Seeger, 1981, 2001; Butt, 2007; Argon, 2008). The most common models either assume a specific functional shape for the Peierls barrier or analytically fit a model to experimental measurements of temperature and strain rate dependent flow stresses (Kocks et al., 1975). Although various non-Schmid models and temperature/strain rate dependent analytical models succeeded in reproducing observed mechanical behaviors in various BCC metals, only a couple of works have combined both effects by assuming a given shape for the Peierls barrier (Gröger et al., 2008c; Weinberger et al., 2012). In particular, no previous work has used atomistic calculations to investigate the proper way of incorporating non-Schmid, temperature and strain rate dependent models into a single constitutive law.

In this paper, we propose a crystal plasticity model for BCC $\alpha$-Fe based on a model recently 
used to successfully predict deformation of Ta ogliocrystals at room temperature (Lim et al., 2015, 2014). The model is further developed by connecting fully atomistic models that incorporate the non-Schmid stress dependent effects of slip with the temperature and strain rate effects. An atomistic potential for BCC Fe that captures the stable compact core dislocation core structure and single peak Peierls barrier (i.e. no metastable split core) consistent with what is predicted from DFT calculations is adopted. Atomistic simulations are conducted to evaluate the critical resolved shear stress (CRSS) for different stress states and quantify non-Schmid effects. The activation enthalpy model incorporating non-Schmid effects as well as temperature and strain rate is developed from atomistic calculations and compared with various analytical models and single crystal experimental data. Our proposed material model captures all of the relevant atomistic effects and is implemented into a CP-FE model to simulate plastic deformations of single and polycrystalline Fe.

\section{BCC Plasticity Model}

Crystal plasticity models assume that the yield occurs through the slip of dislocations on crystallographically defined slip systems. Schmid's law relates applied stress with the shear stress resolved onto each slip system. The criterion for slip to occur on a slip system $\alpha$, is

$$
\tau_{R}=\mathbf{P}_{S}^{\alpha}: \boldsymbol{\sigma}
$$

Here, $\tau_{R}$ is the resistance to slip, $\boldsymbol{\sigma}$ is the applied stress tensor, and $\mathbf{P}_{S}^{\alpha}$ is the Schmid tensor that projects the stress tensor onto the $\alpha$-th slip system. The Schmid tensor can be represented by

$$
\mathbf{P}_{S}^{\alpha}=\frac{1}{2}\left(\mathbf{m}^{\alpha} \otimes \mathbf{n}^{\alpha}+\mathbf{n}^{\alpha} \otimes \mathbf{m}^{\alpha}\right)
$$

where $\mathbf{m}^{\alpha}$ and $\mathbf{n}^{\alpha}$ are the slip direction and slip plane normal of the $\alpha$-th slip system, respectively. While some close-packed metals follow Schmid's law, many BCC metals exhibit yield behavior that deviates from this simple relationship, i.e. tension-compression asymmetry. Non-Schmid effects can be modeled with an alternate slip criterion that additionally depends on stresses that are not resolved onto the $\alpha$-th slip system (Weinberger et al., 2012).

$$
\tau_{c r}=\mathbf{P}_{t o t}^{\alpha}: \boldsymbol{\sigma}=\mathbf{P}_{S}^{\alpha}: \boldsymbol{\sigma}+\mathbf{P}_{n S}^{\alpha}: \boldsymbol{\sigma}
$$


Here, $\mathbf{P}_{t o t}^{\alpha}$ is the total stress projection tensor, $\mathbf{P}_{n S}^{\alpha}$ is the non-Schmid part of the stress projection tensor, and $\tau_{c r}$ is a material constant. Multiple models have been introduced and used for $\mathbf{P}_{n S}^{\alpha}$ (Gröger et al., 2008b; Yalcinkaya et al., 2008), which rely on different non-Schmid stress components. A comparison of the different models and how they are related to each other can be found in work by Lim et al. (Lim et al., 2013). For this work, we selected a generalized form of $\mathbf{P}_{n S}^{\alpha}$ using three orthogonal axes (Yalcinkaya et al., 2008; Lim et al., 2013):

$$
\mathbf{P}_{n S}^{\alpha}=c_{1} \mathbf{P}_{n S}^{\alpha, t m}+c_{2} \mathbf{P}_{n S}^{\alpha, t n}+c_{3} \mathbf{P}_{n S}^{\alpha, n n}+c_{4} \mathbf{P}_{n S}^{\alpha, t t}+c_{5} \mathbf{P}_{n S}^{\alpha, m m}
$$

Here, non-Schmid stress projection tensors are represented as $\mathbf{P}_{n S}^{\alpha, u v}=\mathbf{u}^{\alpha} \otimes \mathbf{v}^{\alpha}$ where $\mathbf{m}^{\alpha}, \mathbf{n}^{\alpha}$, and $\mathbf{t}^{\alpha}=\mathbf{m}^{\alpha} \times \mathbf{n}^{\alpha}$ are unit vectors for the $\alpha$-th slip-system and the $c_{i}$ terms are the non-Schmid material constants that determine the effects of each non-Schmid stress components. In this work, $\mathbf{m}, \mathbf{n}$ and $\mathbf{t}$ correspond to $\langle 111\rangle,\langle 110\rangle$ and $\langle 112\rangle$ crystallographic directions for the BCC crystal, respectively. The non-Schmid constants can be obtained from atomistic simulations (Gröger et al., 2008b) or single crystal experiments (Lim et al., 2013).

The model for $\mathbf{P}_{n S}^{\alpha}$ in Equation (4) is chosen here due to a number of key features. The model captures non-Schmid effects associated with changes in the shear stress parallel to the slip direction, shear stresses perpendicular to the slip direction, and uniaxial tension and compression stresses. The formula for the stress projection tensor relies on three orthogonal directions, thus allowing for the effects of the different shear and normal stresses to be easily interpreted. This has the additional benefit of making the model general to any crystal system.

For the thermally activated deformation mechanisms, the temperature and shear strain rate of the $\alpha$-th slip system can be related to the activation enthalpy, $\Delta H$, using the Arrhenius expression as follows:

$$
\dot{\gamma}^{\alpha}=\dot{\gamma}_{0} \exp \left(-\frac{\Delta H}{k_{B} T}\right)
$$

Here, $\dot{\gamma}^{\alpha}$ is the shear strain rate on $\alpha$-th slip system, $T$ is the temperature, $\dot{\gamma}_{0}$ is a reference strain rate and $k_{B}$ is the Boltzmann constant. Note that $\Delta H$ is a function of the applied stress state and expressions for its stress dependence can be derived in two temperature/ stress regimes using 
dislocation kink-pair theory (Seeger, 1981, 2001; Butt, 2007; Argon, 2008). In the low temperature/ high stress regime, dislocation kinks are modeled as a bulge in the dislocation line from one low energy position to the next by overcoming the local energy maximum. The resulting model, called the Line Tension (LT) model, derives the activation enthalpy from the line tension of the bulge and the shape of the Peierls potential. At the high temperature/ low stress regime in BCC materials, dislocation kinks are modeled as being fully formed and well separated. The activation enthalpy is obtained by assuming that there is only long range elastic interaction between the kinks. This model is referred to as the Elastic Interaction (EI) model. The key difference between the EI and LT models is that the EI model predicts the activation enthalpy to be independent of the Peierls potential and the stress orientation.

Solutions for either the EI and LT activation enthalpy models are typically represented with the following functional form (Kocks et al., 1975):

$$
\Delta H=H_{0}\left[1-\Theta^{p}\right]^{q}
$$

where, $\Theta=\tau / \tau_{R}, \tau$ is the resolved shear stress (RSS), $H_{0}$ is the energy barrier and the values of $p$ and $q$ determine the shape of the energy barrier profile such that $0 \leq p<1$ and $1 \leq q<2$ are satisfied. In terms of the analytical solutions, $p=1$ and $q=2$ corresponds to the LT model for the antiparabolic Peierls potential, $p=4 / 5$ and $q=5 / 4$ approximate the LT models for the sinusoidal and Eshelby potentials, and $p=1 / 2$ and $q=1$ matches the solution for the EI model. Figure 1 shows the most common Peierls potential models; sinusoidal, Eshelby and antiparabolic representations of the Peierls potentials.

Together, Equations (5) and (6) provide a simple relationship between $\tau$, temperature and strain rate. However, the classic definition of $\Theta$ enforces Schmid's law. Recent work by Hale, et al. (Hale et al., 2014a) reveals that the non-Schmid effects can be incorporated into the activation enthalpy model by selecting $\Theta$ such that $\Theta=1$ at the critical stress state, i.e. Equation (3). Using molecular dynamics (MD) simulations and nudged elastic band (NEB) calculations, the stress dependent activation enthalpy was directly measured. Various expressions for theta were 


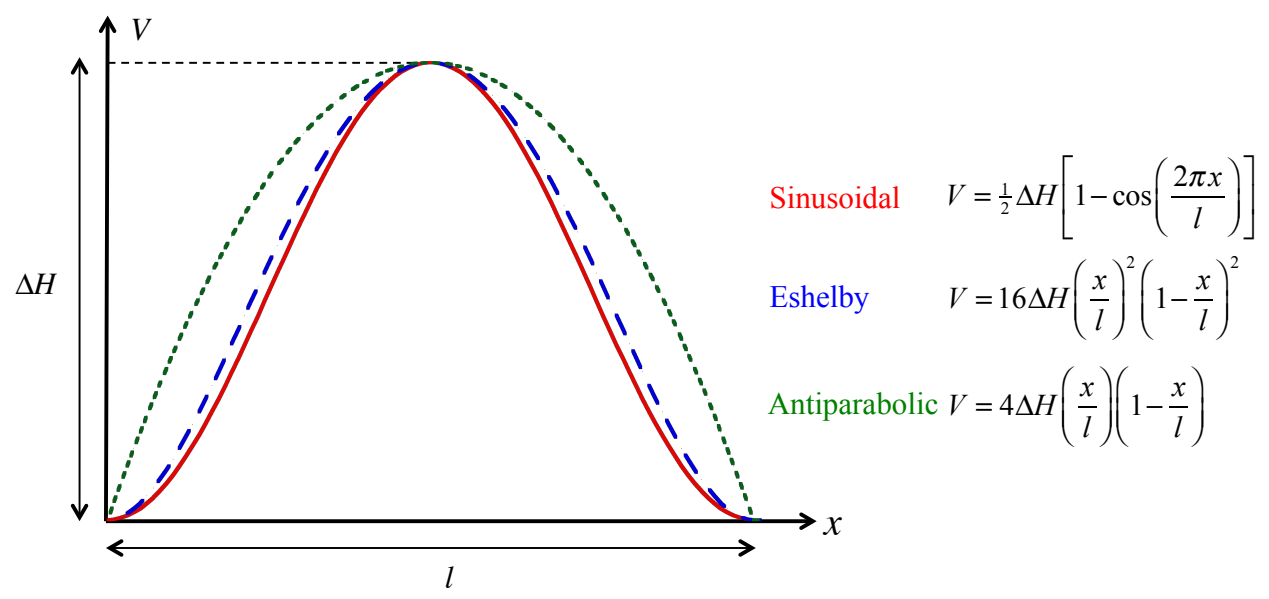

Figure 1: Various functional forms of Peierls potentials: sinusoidal, Eshelby and antiparabolic functions.

investigated, and the following form was found to best normalize the activation enthalpy data.

$$
\Theta=\frac{\tau^{\mathrm{MRSSP}}}{\mathrm{CRSS}}=\frac{\mathbf{P}_{S}: \boldsymbol{\sigma}+c_{1} \mathbf{P}_{n S}^{m t}: \boldsymbol{\sigma}}{\tau_{c r}-c_{2} \mathbf{P}_{n S}^{t n}: \boldsymbol{\sigma}-c_{3} \mathbf{P}_{n S}^{n n}: \boldsymbol{\sigma}-c_{4} \mathbf{P}_{n S}^{t t}: \boldsymbol{\sigma}-c_{5} \mathbf{P}_{n S}^{m m}: \boldsymbol{\sigma}}
$$

Here, $\tau^{\text {MRSSP }}$ represents the shear stress parallel to the slip direction acting on the maximum resolved shear stress plane (MRSSP) and CRSS is the critical resolved shear stress to activate the slip. This expression is notable as the denominator is independent of $\tau^{\mathrm{MRSSP}}$, while the numerator directly depends on it. For systems that obey Schmid's law, $c_{1}=c_{2}=c_{3}=c_{4}=c_{5}=0, \tau_{c r}=\tau_{R}$ and $\Theta=\tau / \tau_{R}$ reducing to Kocks' original model (Kocks et al., 1975). The same reduction can also be obtained by considering stress states where $\mathbf{P}_{S}$ is the only non-zero stress projection tensor in Equation (7).

Using Equations (5) and (6), $\Theta$ can be related to temperature, strain rate and $H_{0}$ as follows:

$$
\Theta=\left(1-\left(\frac{T}{T_{c}}\right)^{1 / q}\right)^{1 / p}, \quad T_{c}=\frac{H_{0}}{k_{B} \ln \left(\dot{\gamma}_{0} / \dot{\gamma}\right)}
$$

Note that Equation (8) provides a simple relation of $\tau$ with temperature and strain rate but does not include non-Schmid effects. Without loss of generality, we can write these equations for the case of uniaxial tension/ compression which exemplifies the interaction between the temperature, 
strain rate and non-Schmid behavior as follows:

$$
\left(1-\left(\frac{T}{T_{c}}\right)^{1 / q}\right)^{1 / p}=\frac{P_{S} \sigma+c_{1} P_{n S}^{m t} \sigma}{\tau_{c r}-c_{2} P_{n S}^{t n} \sigma-c_{3} P_{n S}^{n n} \sigma-c_{4} P_{n S}^{t t} \sigma-c_{5} P_{n S}^{m m} \sigma}
$$

Here, $\sigma=v \cdot \boldsymbol{\sigma} v$ and $P=v \cdot \mathbf{P} v$ where $v$ is the loading direction. Note that $\sigma$ represents a critical level of uniaxial stress to initiate slip that depends on temperature, strain rate and non-Schmid dependence as follows,

$$
\sigma=\frac{B(T, \dot{\gamma}) \tau_{c r}}{P_{S}+c_{1} P_{n S}^{m t}+B(T, \dot{\gamma})\left(c_{2} P_{n S}^{t n}+c_{3} P_{n S}^{n n}+c_{4} P_{n S}^{t t}+c_{5} P_{n S}^{m m}\right)},
$$

where

$$
B(T, \dot{\gamma})=\left(1-\left(\frac{k_{B} T}{H_{0}} \ln \left(\dot{\gamma}_{0} / \dot{\gamma}\right)\right)^{1 / q}\right)^{1 / p}
$$

Equation (10) shows that temperature and strain rate impact the stress needed to induce yield, $\sigma$, through the property $\tau_{c r}$ and several of the non-Schmid projection operators, but not through the Schmid tensor, $P_{S}$, and the term $c_{1} P_{n S}^{m t}$. These temperature and strain rate effects are contained with the function $B(T, \dot{\gamma})$. At high temperature/ low strain rate regime where $B(T, \dot{\gamma})$ is small, $\sigma$ is more dominantly controlled by $P_{S}$ and $c_{1} P_{n S}^{m t}$. At low temperature/ high strain rate regime,

effects of other four non-Schmid contributions, $c_{2} P_{n S}^{t n}, c_{3} P_{n S}^{n n}, c_{4} P_{n S}^{t t}$ and $c_{5} P_{n S}^{m m}$, becomes equally important. Equation (10) can be parameterized to experimental data or atomistic simulations to obtain material parameters, as will be shown in the following sections.

\section{Atomistic Calculations}

In this section, atomistic calculations based on previous work by Hale et al. (Hale et al., 2014b) are used to parameterize the non-Schmid behavior associated with the full range of possible stress states. These calculations are used to determine not only the stress state dependence of the critical stress, but also the stress state dependence of the activation enthalpy. All of the simulations are performed using the LAMMPS molecular dynamics software (Plimpton, 1995). 


\subsection{Interatomic Potential}

The embedded atom method (EAM) potential for Fe developed by Chamati et al. (Chamati et al., 2006) (CPMP potential) is used in this work. It is implemented into LAMMPS using the published functional forms (Chamati et al., 2006) and the potential tables available at the NIST Interatomic Potentials Repository Project web page (Becker et al., 2013). The original paper reports good agreement of the potential's predictions with experimentally measured elastic constants, phonon frequencies, and surface energies. The measured elastic and structural properties agreed well with the published values, with the exception of equilibrium $0 \mathrm{~K}$ lattice parameter of $2.865 \AA$ instead of $2.8665 \AA$ (Figure 2 (a)). The generalized stacking fault has also been calculated for $\{110\} /\langle 111\rangle$ and $\{112\} /\langle 111\rangle$ shearing, revealing unstable stacking fault values of 0.46 and $0.49 \mathrm{eV} / \AA^{2}$, respectively. These values are close to the lower bound reported by DFT calculations (Ventelon and Willaime, 2010).
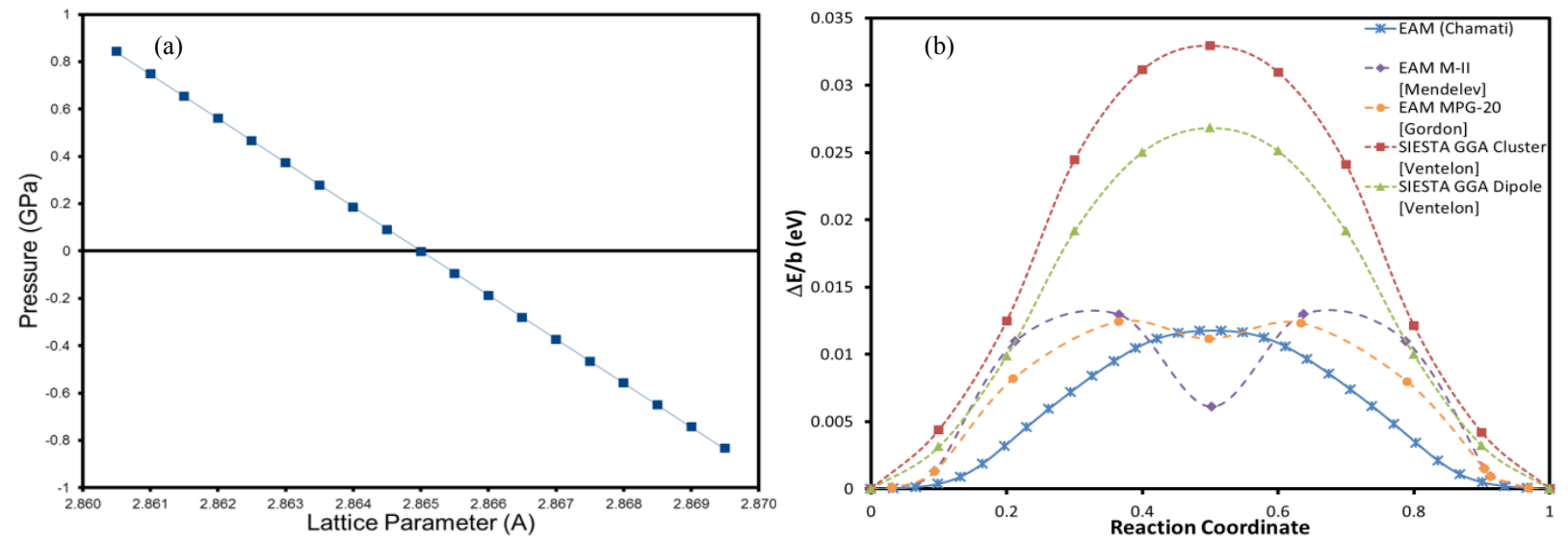

Figure 2: Plots of (a) the measured pressure associated with different lattice parameters at $0 \mathrm{~K}$ and (b) the Peierls energy barriers associated with a $\{110\}$ glide motion of a screw dislocation as predicted by different methods.

The CPMP potential is selected due to the nature of the stable dislocation core and Peierls potential that it predicts. The stable dislocation core is observed to have a non-polarized compact core structure, which is consistent with DFT predictions of the stable core. Calculations of the Peierls barrier reveal a single peak energy pathway associated with the dislocation moving on a $\{110\}$ plane from one stable compact core position to another stable core position one spacing away. Figure 2 (b) shows the Peierls energy curves calculated using the nudged elastic band (NEB) method (Henkelman and Jonsson, 2000; Henkelman et al., 2000) for the CPMP potential, and 
reported for DFT (Ventelon and Willaime, 2007) and two alternate EAM potentials (Gordon et al., 2010, 2011). The CPMP potential and the DFT calculations predict single peak barriers, while the other EAM potentials predict double peak "camel hump" type barriers. The barrier heights for all three EAM potentials are comparable to each other, but are a factor of 2 - 3 times smaller than the DFT results. Preliminary simulations revealed this potential to predict dislocations slip on $\{110\}$ planes for both low $(0.1 \mathrm{~K})$ and ambient temperature simulations.

\subsection{Atomistic Simulation Procedures}

The atomistic simulations are constructed with a perfect BCC block of Fe atoms having crystallographic directions and dimensions of $24 a[\overline{1} 2 \overline{1}] \times 40 a[\overline{1} 01] \times N a[111]$ where $a$ is the lattice constant. Both thin systems, with $N=4$ and containing 46,080 atoms, and thick systems, with $N$ $=48$ and containing 552,960 atoms are constructed. The dislocation is inserted according to the Stroh method for an anisotropic elasticity solution (Stroh, 1958, 1962). The $z$-direction is periodic, while the outer layers of atoms in the $x$ - and $y$-directions are held fixed. Displacement control is used to stress the system by moving the fixed boundary atoms to the strain state associated with the elasticity solution for a particular stress state. The systems are statically relaxed by minimizing the forces acting on the atoms that are not held fixed. This atomic configuration and the orientations of the applied stress, the slip plane and the MRSSP used in the atomic simulations are shown in Figure 3 (Fellinger et al., 2010).
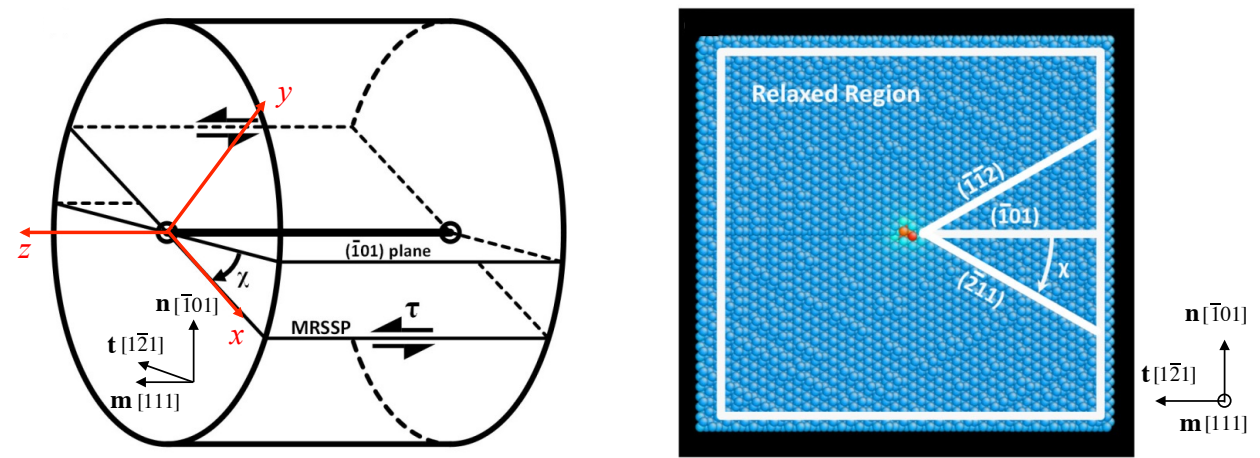

Figure 3: Schematics showing (a) the orientation of the applied stress, the slip plane and the MRSSP and (b) the atomic configuration (Fellinger et al., 2010). The $x y z$-coordinate system in (a) corresponds to stress tensors in Table 1.

Results from the simulations are also used as the end points for the NEB calculations. The NEB calculations take two dislocation core positions subjected to the same applied strain state and 
calculate the energy barrier associated with the low energy pathway for moving from one position to the other. This allows for direct measurement of the activation enthalpy, $\Delta H$, as a function of the stress state. It has been shown that the activation enthalpy obtained from this type of static simulation is consistent with activation enthalpies obtained from dynamic temperature-strain rate simulations (Rodney, 2007). This allows for direct measurement of the activation enthalpy for a given dislocation motion as a function of the stress state. Using NEB permits the energetics associated with 3D kinked dislocation glide to be determined.

The CRSS is obtained by measuring the shear stress acting on the MRSSP in the slip direction that is required to initiate slip. Here, the CRSS is calculated directly by incrementally increasing the applied shear stress acting on the MRSSP, $\tau^{\mathrm{MRSSP}}$, by $0.00005 \times C_{44}=5.7 \mathrm{MPa}$ until the dislocation is observed to move at least one periodic dislocation position. Non-Schmid effects due to shear stresses perpendicular to the slip direction and hydrostatic pressure are evaluated by applying constant perpendicular shear stresses or hydrostatic pressures prior to incrementally increasing $\tau^{\text {MRSSP }}$. CRSS values have also been extrapolated from the NEB calculations by identifying the stress at which the measured energy barrier goes to zero.

The RSS acting on a particular slip system, $\tau^{\alpha}$, can be related to the $\tau^{\text {MRSSP }}$ associated with the same slip direction by

$$
\tau^{\mathrm{MRSSP}}=\frac{\tau^{\alpha}}{\cos \chi}
$$

where $\chi$ is the angle between the MRSSP and the $\{110\}$ slip plane associated with $\alpha$-th slip system. The symmetry associated with the $\langle 111\rangle$ crystallographic projection for BCC crystals allows one to only investigate $-30^{\circ} \leq \chi \leq 30^{\circ}$ as other values of $\chi$ result in other equivalent slip systems having a larger shear stress acting on them. By convention, $\chi=-30^{\circ}$ is associated with applying the stress in the twinning direction of the $\{112\}$ plane, while $\chi=30^{\circ}$ is associated with the anti-twinning direction of the $\{112\}$ plane. All of the simulations performed with this potential reveal the first dislocation glide motion to occur on the $\{110\}$ slip plane with the highest resolved shear stress, thus the values of CRSS in the explored regime are all associated with the same slip system. 


\subsection{Calculations of CRSS and Best-fit $\tau_{c r}$ and non-Schmid Constants}

In order to fit non-Schmid constants in Equation (3), CRSS values are calculated for a number of different stress tensors, $\boldsymbol{\sigma}$. The stress tensors used here and their associated CRSS expressions are listed in Table 1. (Derivations of CRSS criteria can be found in Appendix). These stress tensors are applied in $x y z$-coordinate system in Figure 3 (a). Within the stress tensors, specific components are identified associated with three independent pure shear components $\left(\tau^{\mathrm{MRSSP}}, \tau_{1}^{\perp}\right.$ and $\left.\tau_{2}^{\perp}\right)$ and the hydrostatic pressure $(P)$. CRSS simulations are performed for a variety of $\chi$ angles using each of these stress tensors allowing the constants $\tau_{c r}$ and $c_{i}$ to be fitted to a wide variety of stress states.

Table 1: The stress tensors and the associated criterions used to obtain $\tau_{c r}$ and non-Schmid constants. The stress tensors follow the $x y z$-coordinate system in Figure 3 (a).

\begin{tabular}{ccc}
\hline $\boldsymbol{\sigma}_{\tau_{\mathrm{MRSSP}}}=\left[\begin{array}{ccc}0 & 0 & 0 \\
0 & 0 & \tau^{\mathrm{MRSSP}} \\
0 & \tau^{\mathrm{MRSSP}} & 0\end{array}\right]$ & CRSS criterion \\
\hline $\boldsymbol{\sigma}_{\tau_{\mathrm{MRSSP}, \tau_{1}^{\perp}}}=\left[\begin{array}{ccc}-\tau_{1}^{\perp} & 0 & 0 \\
0 & \tau_{1}^{\perp} & \tau^{\mathrm{MRSSP}} \\
0 & \tau^{\mathrm{MRSSP}} & 0\end{array}\right]$ & $C R S S=\frac{\tau_{c r}-\tau_{1}^{\perp}\left(-c_{2} \sin 2 \chi+\left(c_{3}-c_{4}\right) \cos 2 \chi\right)}{\cos \chi-c_{1} \sin \chi}$ \\
$\boldsymbol{\sigma}_{\tau_{\mathrm{MRSPP}}, \tau_{2}^{\perp}}=\left[\begin{array}{ccc}0 & 0 & 0 \\
0 & \tau_{2}^{\perp} & \tau^{\mathrm{MRSSP}} \\
0 & \tau^{\mathrm{MRSSP}} & -\tau_{2}^{\perp}\end{array}\right]$ & $C R S S=\frac{\tau_{c r}-\tau_{2}^{\perp}\left(-c_{2} \sin \chi \cos \chi+\left(c_{3}-c_{4}\right) \cos ^{2} \chi+\left(c_{4}-c_{5}\right)\right)}{\cos \chi-c_{1} \sin \chi}$ \\
$\boldsymbol{\sigma}_{\tau_{\mathrm{MRSSP}, P}}=$ & {$\left[\begin{array}{ccc}P & 0 & 0 \\
0 & P & \tau^{\mathrm{MRSSP}} \\
0 & \tau^{\mathrm{MRSSP}} & P\end{array}\right] \quad C R S S=\frac{\tau_{c r}+P\left(c_{3}+c_{4}+c_{5}\right)}{\cos \chi-c_{1} \sin \chi}$}
\end{tabular}

By examining the CRSS expressions for the stress tensors containing $\tau_{1}^{\perp}$ and $\tau_{2}^{\perp}, c_{3}, c_{4}$, and $c_{5}$ cannot be independently determined from the shear stresses alone. $\operatorname{CRSS}\left(\chi, \tau_{1}^{\perp}\right)$ depends on $\left(c_{3}-c_{4}\right)$ and $\operatorname{CRSS}\left(\chi, \tau_{2}^{\perp}\right)$ on $\left(c_{3}-c_{4}\right)$ and $\left(c_{4}-c_{5}\right)$. Fitting the constants requires an additional constraint, which is where the pressure dependence comes in. Previous modeling efforts have assumed the hydrostatic effect to be negligible or zero, making $c_{3}+c_{4}+c_{5}=0$ (Gröger et al., 2008b; Lim et al., 2013). Here, we find a mild pressure dependence of CRSS, as shown in Figure 4 (a). The $c_{i}$ constants reflect this dependency.

The values of $\tau_{c r}$ and $\left(c_{3}+c_{4}+c_{5}\right)$ are obtained directly from the pressure dependence, while $c_{1}, c_{2},\left(c_{3}-c_{4}\right)$ and $\left(c_{4}-c_{5}\right)$ are derived from the other simulations by performing a least squares 

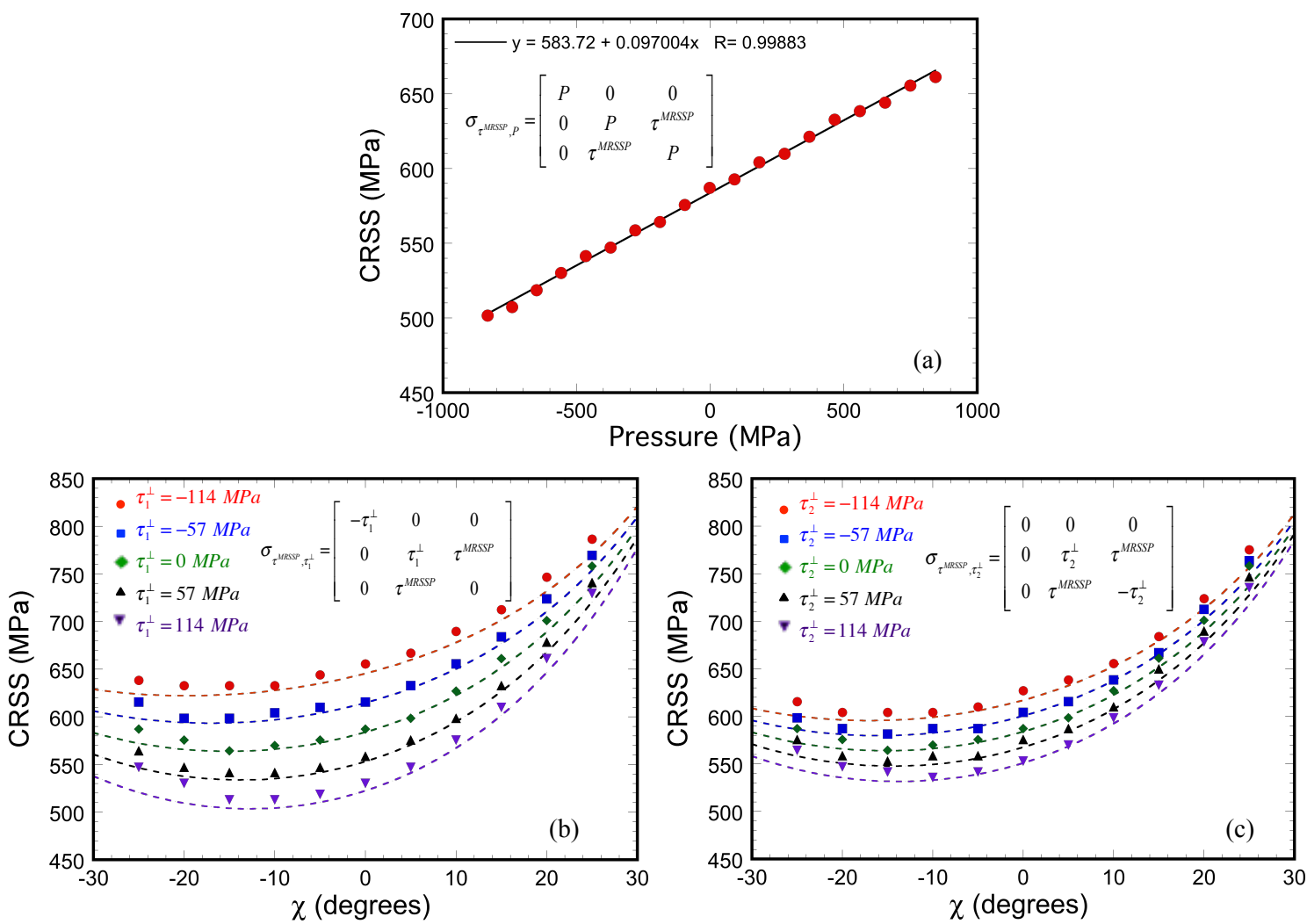

Figure 4: Plots of (a) pressure dependence of the CRSS and (b) CRSS with varying $\tau_{1}^{\perp}$ and (c) $\tau_{2}^{\perp}$ for different $\chi$. The dashed lines in (b) and (c) represent the predicted CRSS using criteria in Table 1.

Table 2: Best-fit non-Schmid constants and $\tau_{c r}$ from atomistic simulations

\begin{tabular}{cccccc}
\hline$c_{1}$ & $c_{2}$ & $c_{3}$ & $c_{4}$ & $c_{5}$ & $\tau_{c r}$ \\
\hline 0.27 & 0.15 & 0.31 & -0.23 & 0.02 & $584 \mathrm{MPa}$ \\
\hline
\end{tabular}

fit to the data associated with $-25^{\circ} \leq \chi \leq 25^{\circ}$ for all $\tau_{1}^{\perp}$ and $\tau_{2}^{\perp}$ explored. Values for $\chi= \pm 30^{\circ}$ are excluded from the fitting as including them was found to skew the data. Table 2 lists the best-fit $\tau_{c r}$ and non-Schmid constants, and Figure 4 (b) and (c) shows simulated and fitted CRSS values at different $\chi$.

In order to investigate the non-Schmid effects on the crystal orientation dependent flow stresses in Fe single crystals, the maximum values of $P_{t o t}^{\alpha}=v \cdot \mathbf{P}_{t o t}^{\alpha} v$ for twenty four $\langle 111\rangle\{110\}$ slip systems associated with uniaxial loadings, $v$, are calculated (Lim et al., 2013). Calculated values of $\max \left(P_{t o t}^{\alpha}\right)$ in tension and compression are the same for the Schmid model ${ }^{1}$, Figure 5 (a), while

\footnotetext{
${ }^{1}$ Hereafter, Schmid model refers to the conventional yield model that follows Schmid's law $\left(\mathbf{P}_{n S}^{\alpha}=0\right)$ and is
} 


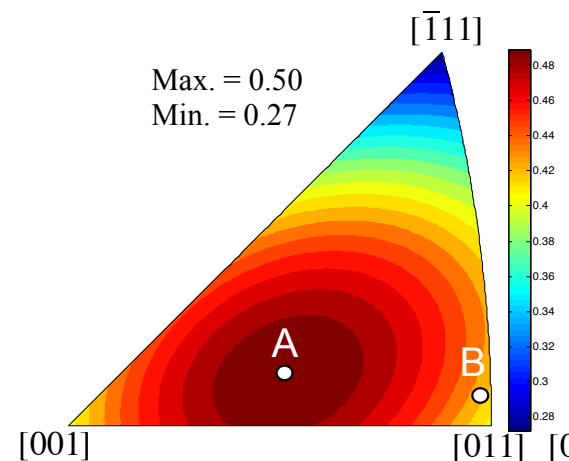

(a) Schmid model (Tension/compression)

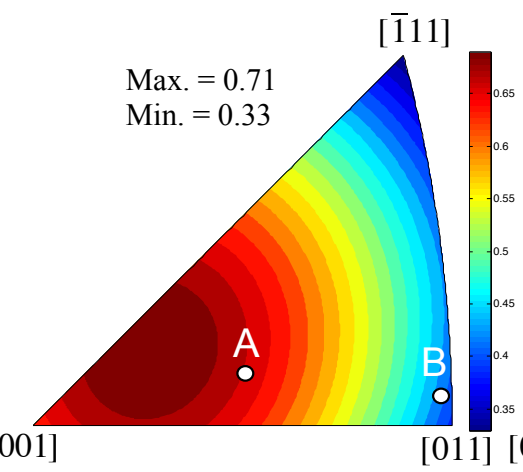

(b) Non-Schmid model (Tension)

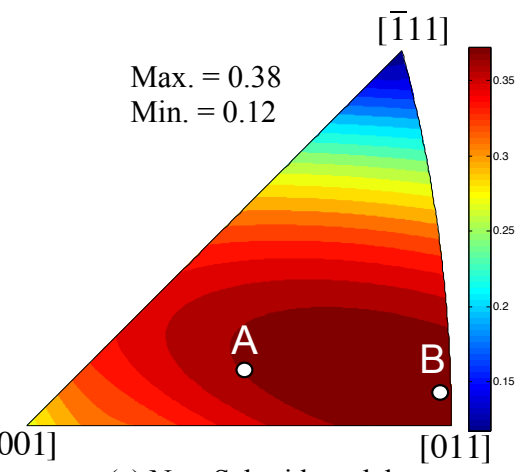

(c) Non-Schmid model (Compression)

Figure 5: The maximum values of $P_{t o t}^{\alpha}$ associated with uniaxial loading within the standard stereographic triangle. (a) Schmid model is tension and compression, (b) non-Schmid model in tension and (c) non-Schmid model in compression.

large tension-compression asymmetry is observed in the non-Schmid model as shown in Figures 5 (b) and (c). The maximum and minimum values of $\max \left(P_{t o t}^{\alpha}\right)$ for the Schmid model are 0.50 and 0.27 at [149] and [111] directions, respectively. For the non-Schmid model, the maximum and the minimum values of $P_{\text {tot }}$ are 0.71 and 0.33 for tension, and 0.38 and 0.12 for compression, respectively.

\subsection{Shear Stress Dependent Peierls Potential}

Figure 6 (a) shows the energy pathway associated with a single dislocation kink glide event at zero applied stress. Figure 6 (b) shows the same corresponding curves with increasing stress for the $\chi=0^{\circ}$ orientation. Extracting good values for $H_{0}$ requires correcting for the slight energy difference between the two end states due to the finite nature of the simulation (Figure 6 (a)). When stress is applied, even small stresses, the work done by the stress overpowers the work associated with changing the core position. This results in the energy maximum being near the first drop-off allowing for the correction to be neglected in measuring $\Delta H$ (Figure 6 (b)). This implies that the activation enthalpy for the stressed systems is associated with the formation of a pair of kinks, and once they form the stress naturally drives them to move the dislocation forward.

Figure 7 shows calculated $\Delta H / H_{0}$ as a function of $\tau^{\mathrm{MRSSP}} / \mathrm{CRSS}$. For comparison, a reported

independent of temperature and strain rate. The non-Schmid model refers to the proposed model incorporating effects of temperature, strain rate and non-Schmid effects using constants in Table 2. 

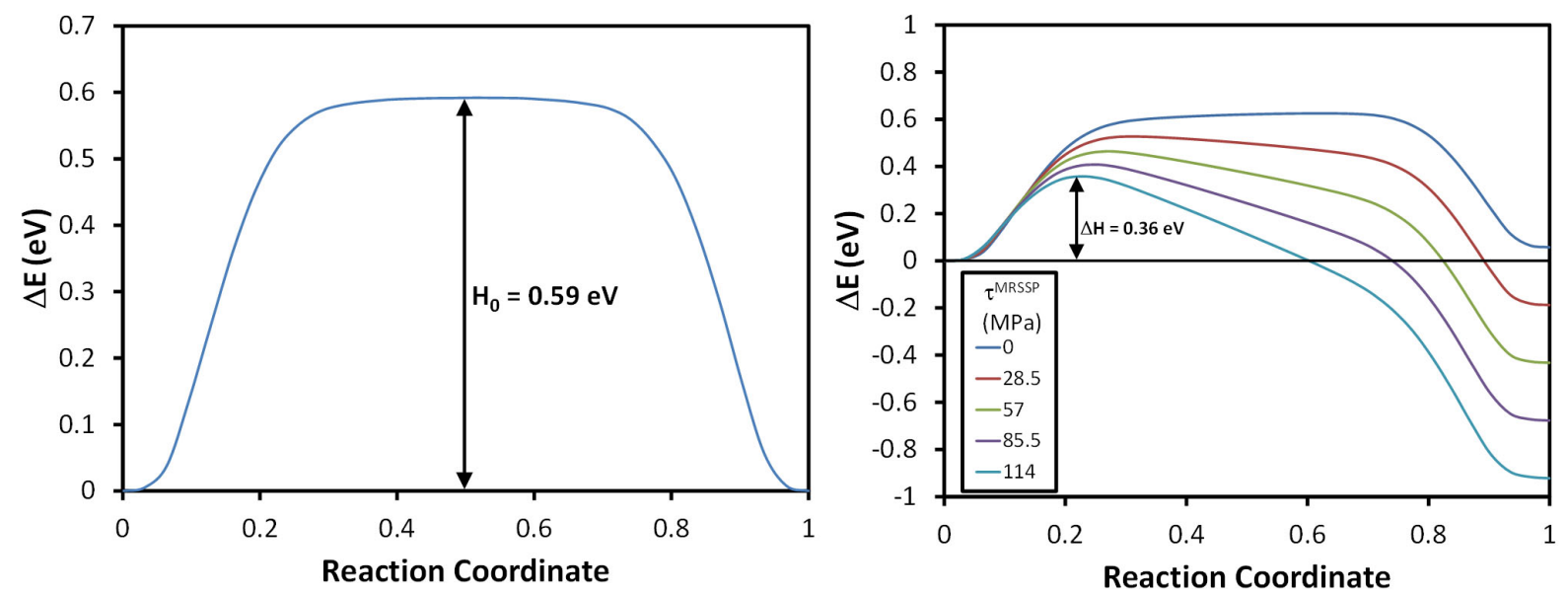

Figure 6: The energy curve associated with the 3D reaction pathway at (a) zero stress and (b) small stresses for $\chi$ $=0$ and $\tau_{1}^{\perp}=\tau_{2}^{\perp}=0$. Note that in (a), an adjustment is applied making the two end state energetically equivalent in order to obtain $H_{0}$.

DFT based line tension model for Fe (Proville et al., 2013) is also included, showing both to be similar, although the MD predicts a slightly smaller $\Delta H / H_{0}$ for a given $\tau^{\mathrm{CRSS}} / \mathrm{CRSS}$ value. When compared to an analytical model, the solution based upon an antiparabolic Peierls potential falls between the MD and DFT curves, and the sinusoidal/Eshelby curve over-predicts $\Delta H$. Empirically fitting Equations (6) and (7) to the data gives a best fit with values of $p=0.9$ and $q=2$, close to the antiparabolic representation of Peierls potential.

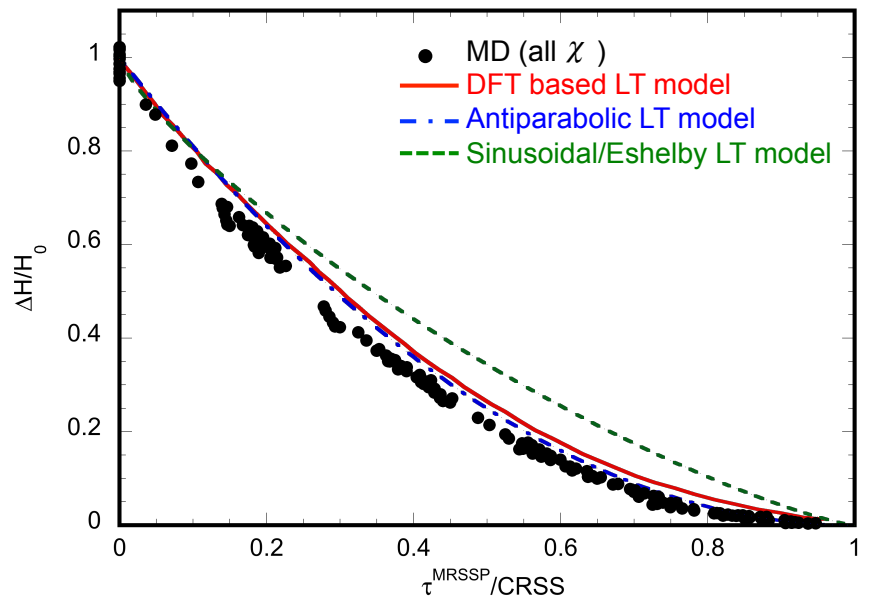

Figure 7: The normalized activation enthalpies for the different stress orientations comparing MD results to DFT (Proville et al., 2013) and analytical LT models adopting antiparabolic and sinusoidal/Eshelby Peierls potentials.

One possible limitation is that the current model is unable to capture the slight variations in 
$\Delta H$ observed with the MD data at $\tau^{\mathrm{MRSSP}}=0$ for different applied perpendicular shears. The resulting scatter reflects the fact that $\Delta H$ is sensitive to the non-Schmid stresses that do not resolve onto the MRSSP, and that no EI (stress independent) region of behavior is observed with the MD results. It should be noted that normalizing the data by $\Delta H / \Delta H\left(\tau^{\mathrm{MRSSP}}=0\right)$ eliminates the scatter indicating that the model could be modified in this fashion. However, the scatter (and lack of EI region) could be due to the finite size of the MD systems. Because of this and the fact that the scatter is small, it is neglected in the development of our model.

\section{Comparison with Experimental Data}

In order to relate Equation (10) to single crystal data obtained from experiments, $T_{c}, c_{i}, p, q$ and $\tau_{c r}$ need to be determined. As shown in Equation (8), $T_{c}$ depends on $H_{0}$ and $\dot{\gamma}$ as well as $\dot{\gamma}_{0}$ that can be estimated from $\dot{\gamma}_{0}=b \rho_{m} w d$, where $b$ is the Burger's vector, $\rho_{m}$ is the mobile dislocation density, $w$ is the attempt frequency and $d$ is the lattice spacing. The value of $T_{c}$ represents the temperature where the thermal part of the flow stress becomes zero for a given strain rate. Thus, instead of calculating $T_{c}$ using $\rho_{m}$ and $w$ which are difficult to obtain directly, we can estimate $T_{c}$ from single crystal data by using the temperature where the thermal part of the flow stress becomes negligible. Values of $p$ and $q$ determine the shapes of the Peierls potential and $\sigma-T$ curve while $\tau_{c r}$ relates $\sigma$ at $0 \mathrm{~K}$. These values can be obtained from atomistic simulations and experimental data. However, the fidelity of the values from the atomistic simulations is still questionable, i.e. this EAM potential predicts a Peierls potential that is about half that predicted from DFT calculations. Furthermore, the disagreement between $\tau_{c r}$ from DFT and experiments has been attributed to quantum effects on lattice vibrations (Barvinschi et al., 2014). Thus, critical information from atomistic results may not be the exact values of $p, q$ and $\tau_{c r}$ but rather an indication of how the Peierls barrier changes under stress. In this regard, $c_{i}$ values as well as the form of Equation (10) obtained from atomistic results are used to parameterize the model.

Based on these assumptions, we adopt $c_{i}$ values from atomistic simulations (Table 2) while $T_{c}$, $\tau_{c r}, p$ and $q$ are obtained from fitting Equation (10) to Fe single crystal experimental data from the literature (Kuramoto et al., 1979a,b). Uniaxial tension tests were conducted using Fe single crystals oriented close to the [149] direction (orientation 'A' in Figure 8 (a)) at various temperatures. Values

of $\sigma$ from experimental data are obtained from $\sigma=\left(\sigma_{y}^{2}-\bar{\sigma}^{2}\right)^{1 / 2}$ where $\sigma_{y}$ is the yield stress and $\bar{\sigma}$ is 
the athermal part of the flow stress $(\bar{\sigma}=18 \mathrm{MPa})$. Note that we use a square root of the sum of the squares decomposition of $\sigma$ opposed to a linear decomposition (Werner, 1987; Hollang et al., 1997, 2001; Brunner, 2000; Seeger and Holzwarth, 2006; Lim et al., 2015, 2014). This decomposition is consistent with the proposed CP-FE model as will be shown in the next section. For the material parameters, we take $H_{0}=0.86 \mathrm{eV}$, which combined with the experimentally determined critical temperature of $T_{c}=350 \mathrm{~K}$ (at a strain rate of $\dot{\gamma}=8 \times 10^{-4} \mathrm{~s}^{-1}$ ) gives $\dot{\gamma}_{0}=1.94 \times 10^{8} \mathrm{~s}^{-1}$. These values are good estimates that will be justified in the following discussion and direct comparisons with experiments.

Three other parameters, $\tau_{c r}, p$ and $q$, are obtained by a least squares fit to temperature dependent single crystal data. For the purpose of comparison, the best-fit values of $\tau_{c r}, p$ and $q$ for both non-Schmid and Schmid models are listed in Table 3. The best-fit $\tau_{c r}$ is larger for the non-Schmid model $\left(\tau_{c r}=542 \mathrm{MPa}\right)$ as compared to the Schmid model $\left(\tau_{c r}=404 \mathrm{MPa}\right)$ due to contributions from the non-Schmid stresses while $p$ and $q$ values are similar for both models. Note that $\tau_{c r}, p$ and $q$ obtained from atomistic simulations are $584 \mathrm{MPa}, 0.9$ and 2, respectively.

Table 3: Best-fit $\tau_{c r}, p$ and $q$ used to reproduce temperature dependent single crystal tensile data for both the non-Schmid and Schmid models.

\begin{tabular}{cccc}
\hline Model & $\tau_{c r}$ & $p$ & $q$ \\
\hline Non-Schmid model & $542 \mathrm{MPa}$ & 0.71 & 1.85 \\
Schmid model & $404 \mathrm{MPa}$ & 0.78 & 1.69 \\
\hline
\end{tabular}

Figure 8 (a) shows measured and fitted flow stresses of Fe single crystal using Equation (10) and parameters listed in Table 3 for tensile flow strengths. Both non-Schmid and Schmid models accurately reproduced measured temperature dependent $\sigma$ of single crystal in orientation 'A'. The non-Schmid model predicts a large tension-compression asymmetry especially at low temperatures/ high stress regimes (dashed line in Figure 8 (a)) which cannot be produced in a Schmid based model. The experiments are only in tension, and thus no tension-compression asymmetry was reported. This is attributed to different $\max \left(P_{\text {tot }}^{\alpha}\right)$ as shown in Figures $5(\mathrm{~b})$ and (c). Tension-compression asymmetry decreases with temperature and becomes negligible at $T>250 \mathrm{~K}$. On the other hand, the Schmid model did not exhibit tension-compression asymmetry, consistent with results in Figure 5 (a).

Figure 8 (b) compares the temperature dependent $\tau / \tau_{R}$ obtained from fitting material param- 

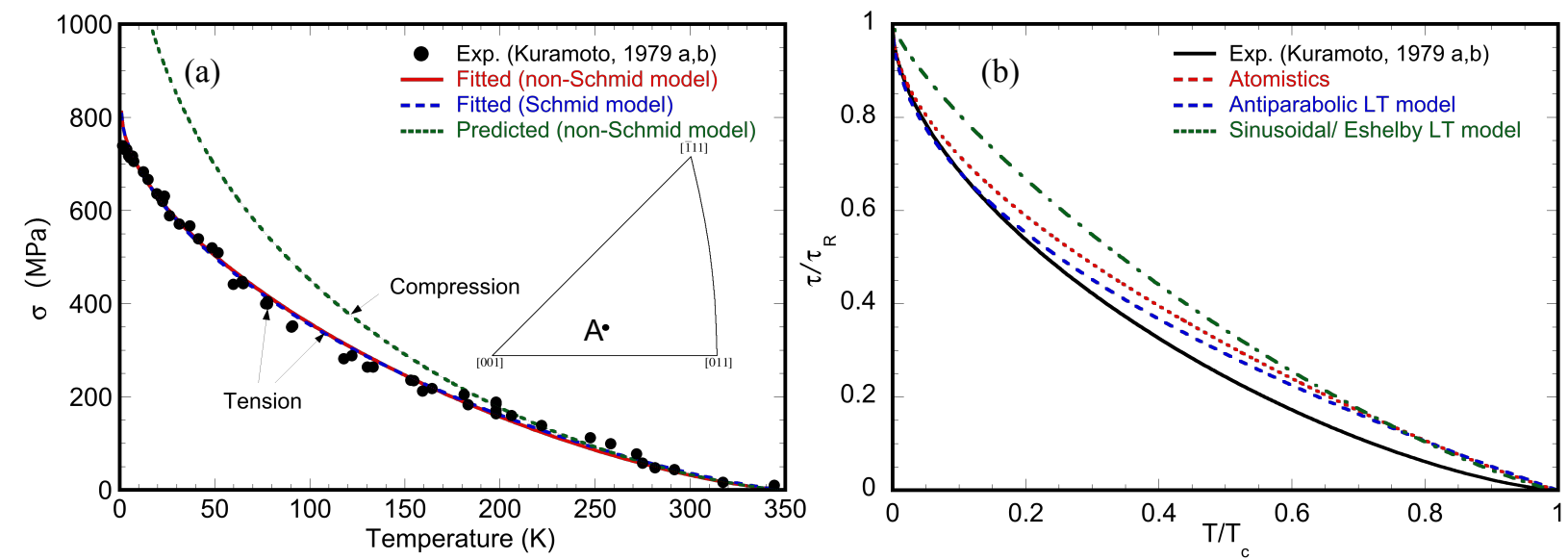

Figure 8: Plots of (a) measured and fitted temperature dependent flow stresses of Fe single crystals for orientation 'A' using non-Schmid and Schmid models. The experiments shown are tensile data only and the Schmid-based model (blue) predicts equal tensile and compressive strengths. The non-Schmid model was fit to the tensile data (red) and predicted in compression (green), exhibiting tension compression asymmetry. (b) $\tau / \tau_{R}$ versus $T / T_{c}$ using fits from experimental data, atomistics and analytical LT models.

eters to the single crystal experiments (Kuramoto et al., 1979a,b), MD simulations and analytical LT models adopting antiparabolic and sinusoidal/Eshelby Peierls potentials. Here, $\tau$ and $T$ are normalized by $\tau_{R}$ and $T_{c}$, respectively, to compare temperature dependence. In the low temperature/ high stress regime, $\tau / \tau_{R}$ from the experimental fit and MD agree well to antiparabolic LT model. At high temperature/ low stress regime, $\tau / \tau_{R}$ from experimental fit deviate from those obtained from atomistic simulations and other analytical LT models.
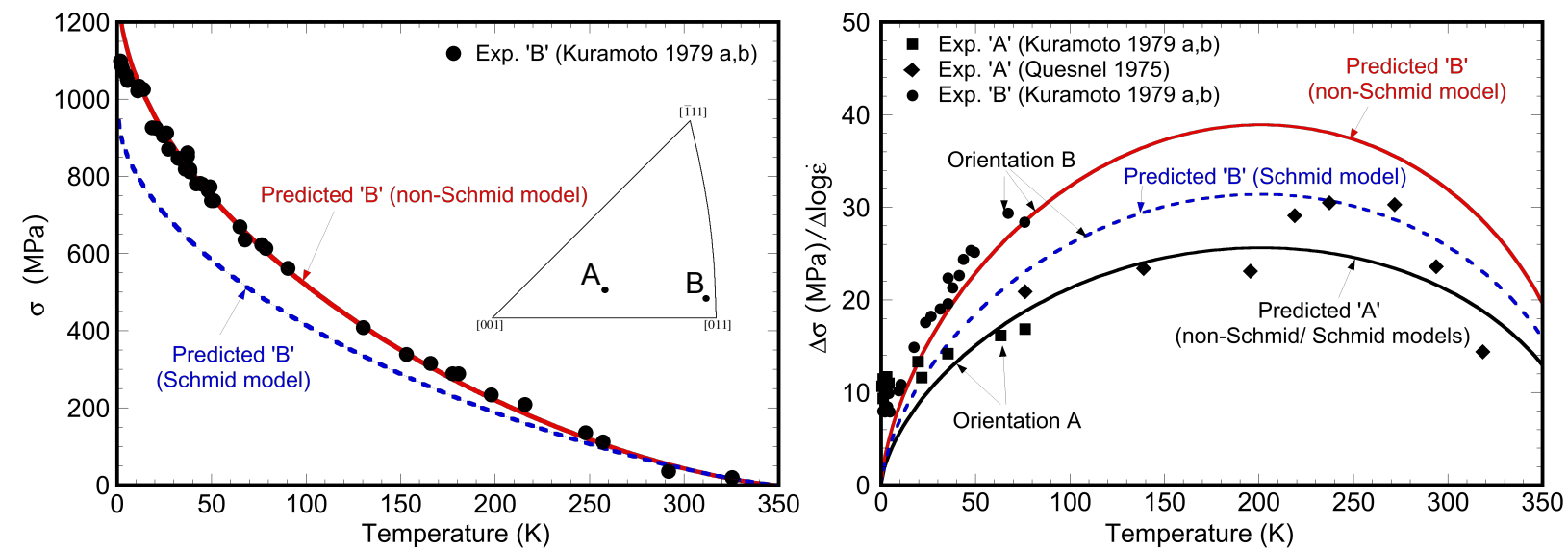

Figure 9: Plots of (a) measured and predicted temperature dependent flow stresses of Fe single crystals for orientation 'B' using non-Schmid and Schmid models and (b) measured and predicted strain rate sensitivity of Fe single crystals in orientations ' $\mathrm{A}$ ' and ' $\mathrm{B}$ '. 
The model parameterized for a single crystal ' $\mathrm{A}$ ' is then used to predict the temperature dependent $\sigma$ for a different crystal orientation, 'B'. Figure 9 (a) shows the predicted $\sigma$ using the non-Schmid (red solid line) and the Schmid (blue dashed line) models in orientation 'B'. Although both models successfully reproduce the temperature dependent $\sigma$ in orientation 'A' (Figure 8 (a)), the non-Schmid model accurately predicts temperature dependent $\sigma$ in orientation 'B'. The Schmid model under-predicted $\sigma$ for all temperature regimes and this clearly demonstrates that it is important to consider non-Schmid stress contributions to accurately predict orientation dependent flow stress.

In order to investigate strain rate dependence, $\Delta \sigma=\sigma\left(\dot{\varepsilon}=8 \times 10^{-4} \mathrm{~s}^{-1}\right)-\sigma\left(\dot{\varepsilon}=8 \times 10^{-5} \mathrm{~s}^{-1}\right)$ is obtained for two crystal orientations. Figure 9 (b) shows measured (Quesnel et al., 1975; Kuramoto et al., 1979a,b) and predicted strain rate sensitivity, $(\Delta \sigma / \Delta \log \dot{\varepsilon})$, as a function of temperature for orientations 'A' and 'B'. Note that both the non-Schmid and Schmid models fit to the $\sigma-T$ plot predict the same strain rate dependence for orientation 'A' (black solid line). Similar to the crystal orientation dependence in Figure 9 (a), the strain rate sensitivity is more accurately predicted by the non-Schmid model while the Schmid-based model under-predicted strain rate dependence.

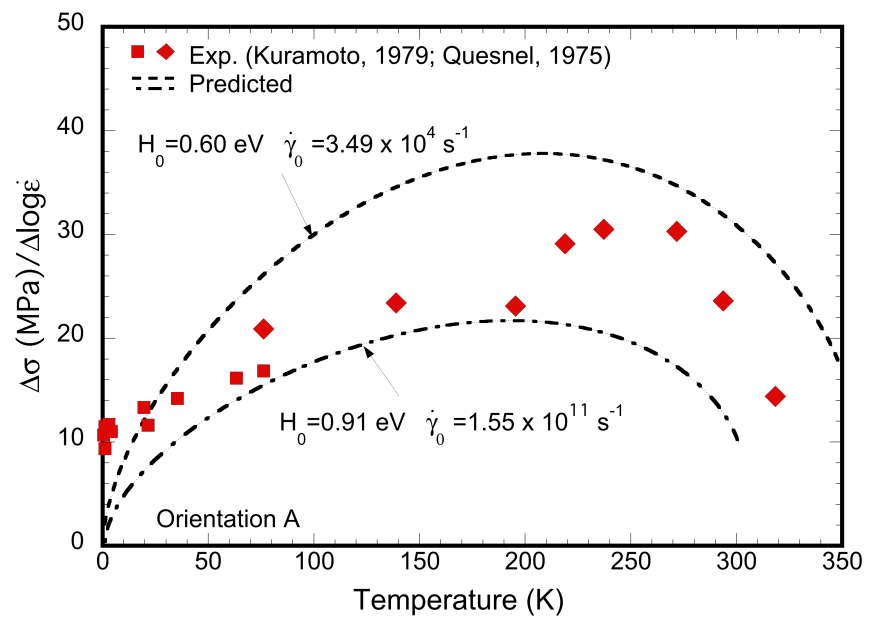

Figure 10: Measured and predicted strain rate sensitivity of Fe single crystals in orientation 'A'. Here, two dashed line show model prediction with different $H_{0}$ and $\dot{\gamma}_{0}$.

The strain rate dependence of $\sigma$ is determined by $H_{0}$ and $\dot{\gamma}_{0}$ values for a given $T_{c}$ using Equations (10) and (11). We have used $H_{0}=0.86 \mathrm{eV}$ obtained from DFT calculations by Proville et al. (Proville et al., 2013) and $\dot{\gamma}_{0}=1.94 \times 10^{8} \mathrm{~s}^{-1}$ from single crystal experiments. Other reported values 
of $H_{0}$ from atomistic simulation in the current work and from the literature range within 0.60 $0.91 \mathrm{eV}$ (Brunner and Diehl, 1991a,b; Proville et al., 2013). Furthermore, we can estimate $T_{c}$ at $\dot{\varepsilon}=8 \times 10^{-5} \mathrm{~s}^{-1}$ to be in the range of $300-350 \mathrm{~K}$ from experimental data (Kuramoto et al., $1979 \mathrm{a}, \mathrm{b})$ that correspond to $3.49 \times 10^{4} \mathrm{~s}^{-1} \leq \dot{\gamma}_{0} \leq 1.55 \times 10^{11} \mathrm{~s}^{-1}$. Using these ranges of values for $\Delta H_{0}$ and $\dot{\gamma}_{0}$, the two dashed lines in Figure 10 denote upper and lower bounds of the rate sensitivity for single crystal in orientation 'A'. It is shown that predictions using the non-Schmid model using reported values of $H_{0}$ and estimate of $T_{c}$ provide reasonable bounds on the measured strain rate sensitivity data.

\section{CP-FE Simulations}

The proposed activation enthalpy model parameterized using atomistic simulations and single crystal experiments successfully predicted the temperature, strain rate and orientation dependent yield behavior of Fe single crystals. In the following section, we implement the model into a BCC crystal plasticity framework to simulate grain-scale deformations of single and polycrystalline Fe.

\subsection{Crystal Plasticity Formulations}

Crystal plasticity - finite element (CP-FE) models allow detailed investigation of grain-scale deformation behavior in polycrystalline metals, e.g. plastic anisotropy and texture evolution. The activation enthalpy model outlined in the previous section is implemented into a BCC CP-FE framework developed at Sandia National Laboratories (Biffle, 1987; Lim et al., 2013, 2015). The foundation of the model is based on well-established Peirce-Asaro-Needleman (PAN) formulations (Peirce et al., 1982). The model uses a multiplicative decomposition of the deformation gradient and assumes that the plastic deformation is caused only by the dislocation slip at each material point (Lee, 1969; Asaro, 1983). The model uses a fully implicit time integration scheme to conduct a quasi-static analysis. Detailed kinematics of the model can be found elsewhere (Peirce et al., 1982; Bronkhorst et al., 1992; Kalidindi et al., 1992).

For a rate dependent crystal plasticity model, the slip rate on $\alpha$-th slip system, $\dot{\gamma}^{\alpha}$, is represented as a power-law function of resolved shear stress, $\tau^{\alpha}$ and slip resistance, $g^{\alpha}$ (Hutchinson, 1976):

$$
\dot{\gamma}^{\alpha}=\dot{\gamma}^{0}\left(\frac{\tau^{\alpha}}{g^{\alpha}}\right)^{1 / m} .
$$


Here, $\dot{\gamma}^{0}$ is the reference shear rate and $m$ is the rate sensitivity factor. In this work, $\dot{\gamma}^{0}=0.001 \mathrm{~s}^{-1}$ and $m=0.04$ are used and assumed to be constant throughout the deformation for all $24\langle 111\rangle\{110\}$ slip systems. Note that the Arrhenius expression in Equation (5) relates the temperature and shear strain rate to the activation enthalpy and used to formulate temperature and strain rate dependent lattice resistance or the thermal part of the flow stress. On the other hand, Equation (13) serves as a numerical device to satisfy mathematical generality in rate dependent crystal plasticity formulations, rather than a physical strain rate. The slip resistance of the $\alpha$-th slip system, $g^{\alpha}$, can be decomposed into $\tau^{* \alpha}$ and $\tau_{o b s}^{\alpha}$ as follows:

$$
g^{\alpha}=\sqrt{\left(\tau^{* \alpha}\right)^{2}+\left(\tau_{o b s}^{\alpha}\right)^{2}}
$$

Here, $\tau^{* \alpha}$ is the thermal part of the flow stress attributed to lattice resistance and $\tau_{o b s}^{\alpha}$ is the strain hardening or athermal contribution that arises from long-range interactions such as forest dislocations. Note that $\tau^{*}$ is generally ignored in close-packed metals and the formulation in Equation (14) successfully describes yield surface transitions between the non-Schmid and Schmid behavior.

Using Equations (7), (8) and (11), an expression for the thermal resistance to flow, $\tau^{*}$, can be obtained by noting that:

$$
B\left(T, \dot{\gamma}^{\alpha}\right)=\frac{\mathbf{P}_{S}: \boldsymbol{\sigma}+c_{1} \mathbf{P}_{n S}^{m t}: \boldsymbol{\sigma}}{\tau_{c r}-c_{2} \mathbf{P}_{n S}^{t n}: \boldsymbol{\sigma}-c_{3} \mathbf{P}_{n S}^{n n}: \boldsymbol{\sigma}-c_{4} \mathbf{P}_{n S}^{t t}: \boldsymbol{\sigma}-c_{5} \mathbf{P}_{n S}^{m m}: \boldsymbol{\sigma}}
$$

Slip occurs when the stress projected onto a slip system exceeds the resistance to flow. The projected stress meets the lattice resistance when $\tau^{* \alpha}=\mathbf{P}_{S}^{\alpha}: \sigma$. For the case where lattice resistance is the only resistance to plastic flow, this criterion describes the onset of yielding according to Equation (13). Combining this expression with Equation (15) (which is simply a definition of flow obtained from Equations (7), (8) and (11)) and rearranging:

$$
\tau^{* \alpha}=B\left(T, \dot{\gamma}^{\alpha}\right)\left[\tau_{c r}-c_{2} \mathbf{P}_{n S}^{\alpha, t n}: \boldsymbol{\sigma}-c_{3} \mathbf{P}_{n S}^{\alpha, n n}: \boldsymbol{\sigma}-c_{4} \mathbf{P}_{n S}^{\alpha, t t}: \boldsymbol{\sigma}-c_{5} \mathbf{P}_{n S}^{\alpha, m m}: \boldsymbol{\sigma}\right]-c_{1} \mathbf{P}_{n S}^{m t}: \boldsymbol{\sigma}
$$

where, $B\left(T, \dot{\gamma}^{\alpha}\right)$ is a temperature and strain rate dependent constant that ranges from $0\left(T=T_{c}\right)$ to $1(T=0 \mathrm{~K})$. Thus, for $0 \leq T \leq T_{c}, \tau_{c r}-\mathbf{P}_{n S}^{\alpha}: \boldsymbol{\sigma} \leq \tau^{* \alpha} \leq-c_{1} \mathbf{P}_{n S}^{\alpha, m t}: \boldsymbol{\sigma}$ is satisfied. 
An obstacle strength, $\tau_{o b s}$, for each slip system is obtained from a dislocation density-based hardening law (Taylor, 1934; Lee et al., 2010).

$$
\tau_{o b s}^{\alpha}=A \mu b \sqrt{\sum_{\beta=1}^{24} \rho^{\beta}} .
$$

Here, $A$ is a material constant, $\mu$ is the shear modulus, $b$ is the Burger's vector, and $\rho^{\beta}$ is the dislocation density on slip system $\beta$. In this work, $A=0.4, \mu=69.7 \mathrm{GPa}$ and $b=2.48 \AA$ are used (Hirth and Lothe, 1982). The evolution of dislocation density for the $\alpha$-th slip system is obtained by a standard phenomenological equation Kocks (1976) as follows:

$$
\dot{\rho}^{\alpha}=\left(\kappa_{1} \sqrt{\sum_{\beta=1}^{\mathrm{NS}} \rho^{\beta}}-\kappa_{2} \rho^{\alpha}\right) \cdot\left|\dot{\gamma}^{\alpha}\right|,
$$

where, $\kappa_{1}$ and $\kappa_{2}$ are hardening parameters that represent generation and annihilation of dislocations, respectively, and determine the shape of the hardening curve. In this work, we assume that hardening parameters are independent of temperature and strain rate. Note that material parameters needed to formulate $\tau^{*}$ in Equation (16) are obtained from atomistic simulations $\left(c_{1}-c_{5}\right)$, single crystal experiments $\left(\tau_{c r}, p\right.$ and $\left.q\right)$ and DFT simulations $\left(H_{0}\right)$. The two hardening parameters, $\kappa_{1}$ and $\kappa_{2}$, represent the evolution of $\tau_{o b s}$ in Equation (17) and are parameterized from the stress-strain responses of single crystal Fe at $T=348 \mathrm{~K}$ and $\dot{\epsilon}=8 \times 10^{-5}$ in Figure 11 (a) (Kuramoto et al., 1979a,b) which results in values of $\kappa_{1}=7 \times 10^{5} \mathrm{~m}^{-1}$ and $\kappa_{2}=50$.

\subsection{Single Crystal Deformation}

The atomistically-informed CP-FE model is used to simulate uniaxial tension of Fe single crystals for various temperatures and strain rates. In this work, eight-noded hexahedral finite elements having a single integration point at the element centroid are used. A single finite element is loaded

along orientation 'A', close to [149] direction (Figure 5) with (a) varying temperature at $\dot{\varepsilon}=8 \times 10^{-5}$ $\mathrm{s}^{-1}$ and (b) varying strain rate at $T=300 \mathrm{~K}$. Note that the strain rate is controlled by the simulation time and the applied strain (i.e. 1250 seconds for $10 \%$ deformation) and the temperature is assumed to be constant throughout the simulation. Figures 11 (a) compares measured (Kuramoto et al., 1979a) and predicted stress-strain responses of Fe single crystal at six temperatures, from 
$4 \mathrm{~K}$ to $348 \mathrm{~K}$. The predicted temperature dependent stress-strain responses agree well with the experimental data. In particular, the model accurately captures the large temperature dependence of yield while no significant temperature dependent strain hardening behavior is observed. Figure 11 (b) shows predicted strain rate dependence at $300 \mathrm{~K}$. Varying strain rates by six orders of magnitude from $10^{-4} \mathrm{~s}^{-1}$ to $10^{2} \mathrm{~s}^{-1}$, predicted yield stresses increased by more than a factor of ten, while no significant difference in strain hardening is observed.
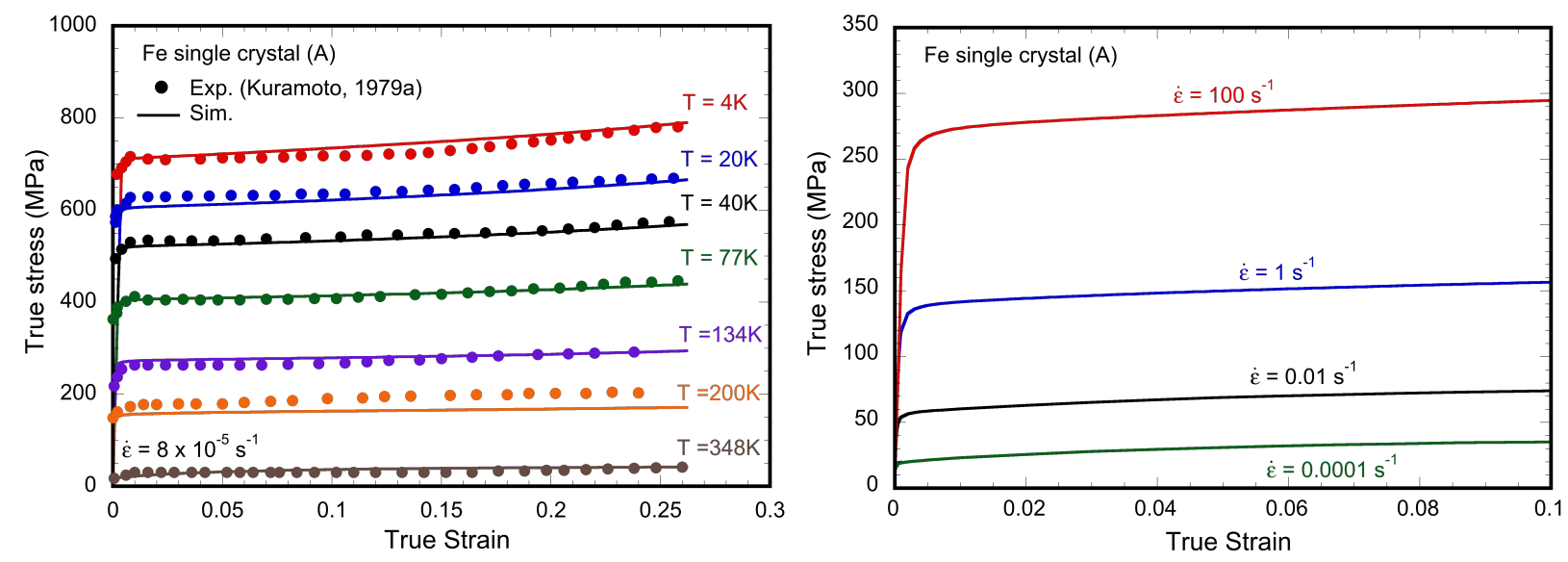

Figure 11: Plots of (a) measured and predicted temperature dependence and (b) predicted strain rate dependence of Fe single crystals in [149] loading direction (orientation 'A')

In order to investigate non-Schmid effect in single crystal deformation, biaxial yield surfaces are constructed using Equation (10) for different temperatures (0, 150 and $300 \mathrm{~K}$ ) at a constant strain rate of $10^{-4} \mathrm{~s}^{-1}$. Figure 12 shows the predicted biaxial yield surfaces of single crystals using the non-Schmid and Schmid model in $[001] /[0 \overline{1} 0],[011] /[01 \overline{1}]$ and $[\overline{1} 11] /[01 \overline{1}]$ directions. For each axis, $\sigma_{11}$ and $\sigma_{22}$ are normalized by the yield stress in $\sigma_{11}$ direction $\left(\sigma_{y 1}\right)$. These results show that, as expected, the non-Schmid effect is more dominant at low temperatures as exhibited by the deviations from the Schmid-based yield surfaces. Notably, the tension-compression asymmetry decreases with temperature in the non-Schmid Model. Predicted biaxial yield surfaces from the Schmid model do not exhibit tension-compression asymmetry and temperature dependence.

The tension-compression asymmetry can be quantified with a strength differential (SD), defined as (Gröger et al., 2008b):

$$
\mathrm{SD}=\frac{\sigma_{T}-\sigma_{C}}{\left(\sigma_{T}+\sigma_{C}\right) / 2}
$$






Figure 12: Temperature dependent biaxial yield surfaces for Fe single crystals in $[001] /[0 \overline{1} 0],[011] /[01 \overline{1}]$ and $[\overline{1} 11] /[01 \overline{1}]$ directions.

Here, $\sigma_{T}$ and $\sigma_{C}$ are $\sigma$ in tension and compression, respectively. For the Schmid model which exhibits no tension-compression symmetry, the SD value is zero while a positive SD corresponds to $\sigma_{T}>\sigma_{C}$ and negative SD denotes $\sigma_{T}<\sigma_{C}$.

Figures 13 (a) - (c) show predicted $\sigma_{T}, \sigma_{C}$ and SD at temperatures of $0 \mathrm{~K}, 150 \mathrm{~K}$ and 300 $\mathrm{K}$, using Equation (10). For all temperatures, the minimum and the maximum $\sigma$ are observed near the [001] and [111] poles, respectively. Note that predicted $\mathrm{SD}$ at $T=0 \mathrm{~K}$ and $150 \mathrm{~K}$ is negative $\left(\sigma_{T}<\sigma_{C}\right)$ in all crystal orientations while at $T=300 \mathrm{~K}$, positive values of $\mathrm{SD}\left(\sigma_{T}>\sigma_{C}\right)$ are predicted near the $[011]$ pole. This result shows that the model predicts crystal orientation dependent yield behaviors that deviate from the Schmid model as well as temperature dependent tension-compression asymmetry.

Using the CP-FE model, the crystal rotations of Fe single crystals are predicted upon uniaxial loading. In order to closely reproduce isochoric deformation boundary conditions in tension and compression (Buchheit et al., 2005), $\varepsilon_{y y}=\varepsilon_{z z}=-0.25$ and $\varepsilon_{y y}=\varepsilon_{z z}=0.25\left(\dot{\varepsilon}_{y y} / \dot{\varepsilon}_{x x}=\dot{\varepsilon}_{z z} / \dot{\varepsilon}_{x x}=\right.$ -0.5 ) are applied to a single finite element, respectively. Figure 14 compares crystal rotations during uniaxial tension and compression up to around $50 \%$ applied strain at different temperatures, $100 \mathrm{~K}, 200 \mathrm{~K}$ and $300 \mathrm{~K}$. The model predicts changes in the texture evolution for different temperatures with more pronounced deviation near the [001] pole. Note that the influence of the strain rate on the deformation of Fe single crystals, i.e. yield surfaces, tension-compression asymmetry and crystal rotations, can be estimated from Equation (11), i.e. $T$ is inversely proportional 




(a) $\sigma$ (Tension)

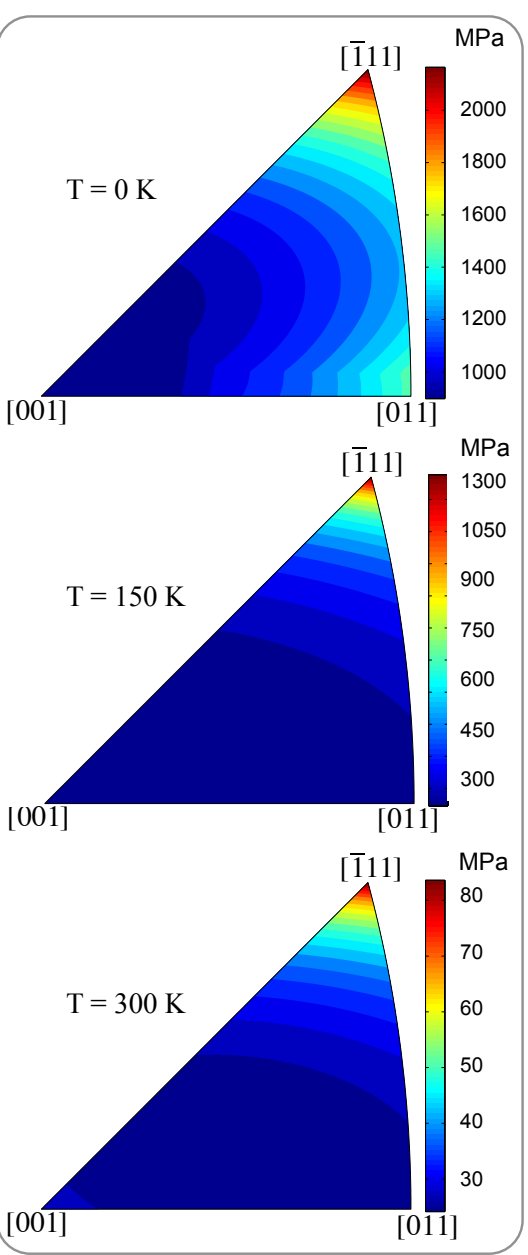

(b) $\sigma$ (Compression)

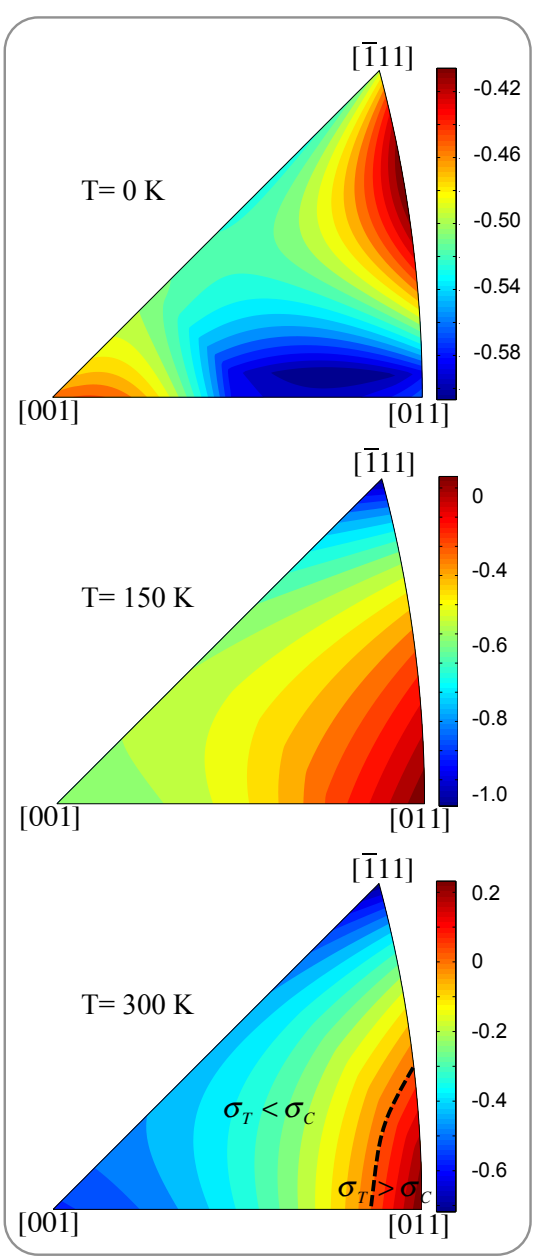

(c) SD

Figure 13: Predicted $\sigma(\mathrm{MPa})$ in tension and compression, and strength differential (SD) of Fe single crystals at 0 K, $150 \mathrm{~K}$ and $300 \mathrm{~K}$.

to $\ln \left(\dot{\gamma}_{0} / \dot{\gamma}^{\alpha}\right)$.

\subsection{Polycrystal Deformation}

In order to simulate deformation of polycrystalline Fe, a three-dimensional cubic specimen with 139 equiaxed grains is constructed using the DREAM.3-D software package developed at the Air Force Research Laboratory (AFRL) with contributions from Carnegie Mellon University (Saylor et al., 2004; Groeber et al., 2008a,b) as shown in Figure 15 (a). A total of 50 $=125,000$ hexahedral elements are used and each grain is assigned a random crystal orientation. Uniaxial tension of polycrystalline Fe for various temperatures at a nominal strain rate of $10^{-4} \mathrm{~s}^{-1}$ are simulated using material parameters used in single crystal simulations. As shown in Figure 15 (b), large 


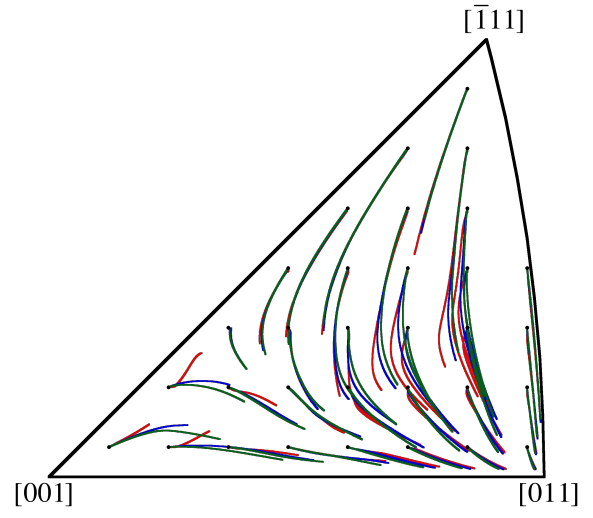

(a) Tension

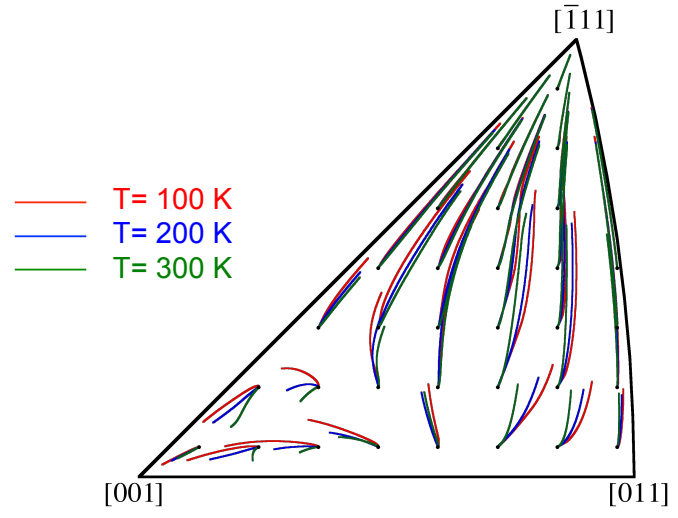

(b) Compression

Figure 14: Inverse pole figures of the tensile axis as it rotates in the standard unit triangle during plastic deformation at $100 \mathrm{~K}, 200 \mathrm{~K}$ and $300 \mathrm{~K}$ for tension and compression.

temperature dependent initial yield is predicted by the model while the strain hardening was less affected by the temperature similar to the single crystal behavior.

Figure 16 (a) shows predicted biaxial yield surfaces of polycrystalline Fe using a 3D polycrystalline mesh at various temperatures. It is shown that CP-FE predictions of polycrystalline yield surfaces deviate from isotropic yield functions, especially at low temperatures. As temperature is raised above $100 \mathrm{~K}$, the predicted yield surface quickly converges to standard isotropic yield functions, e.g. Tresca or Von Mises yield functions.

Various yield surfaces have been proposed to describe anisotropic yield behaviors of polycrystalline metals. For example, Drucker-Prager yield surface (Drucker and Prager, 1952) is capable of capturing tension-compression asymmetry and pressure dependence while some yield functions use second and third invariants of the deviatoric stress to represent the $J_{3}$ dependence of flow (Drucker, 1949; Cazacu and Barlat, 2004). Based on these yield criteria, we can use the following yield law that incorporates both non-Schmid effect and the pressure dependence:

$$
f\left(I_{1}, J_{2}, J_{3}\right)=\alpha_{1} I_{1}+\left(J_{2}^{3 / 2}+\alpha_{2} J_{3}\right)^{1 / 3}
$$

Here, $I_{1}$ is the first stress invariant and $J_{2}$ and $J_{3}$ are the second and third invariants of the deviatoric stress, respectively. $\alpha_{1}$ represents the magnitude of the pressure dependence while $\alpha_{2}$ determines the shape of the yield surface in the deviatoric plane. Thus, $\alpha_{1}=\alpha_{2}=0$ in Equation (20) reduces it to the von Mises yield surface. 


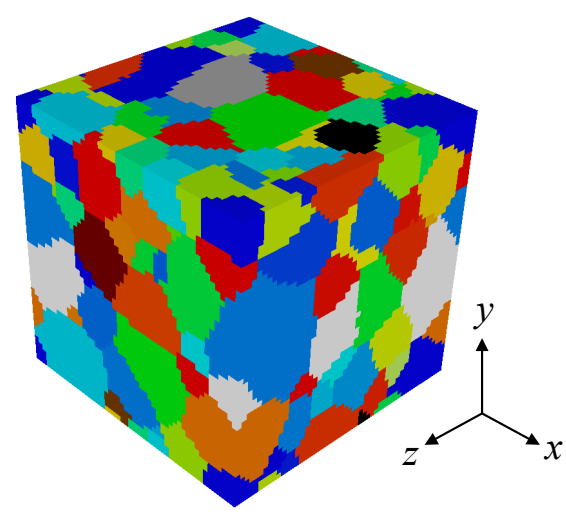

(a) 3-D FE model of Fe polycrystal

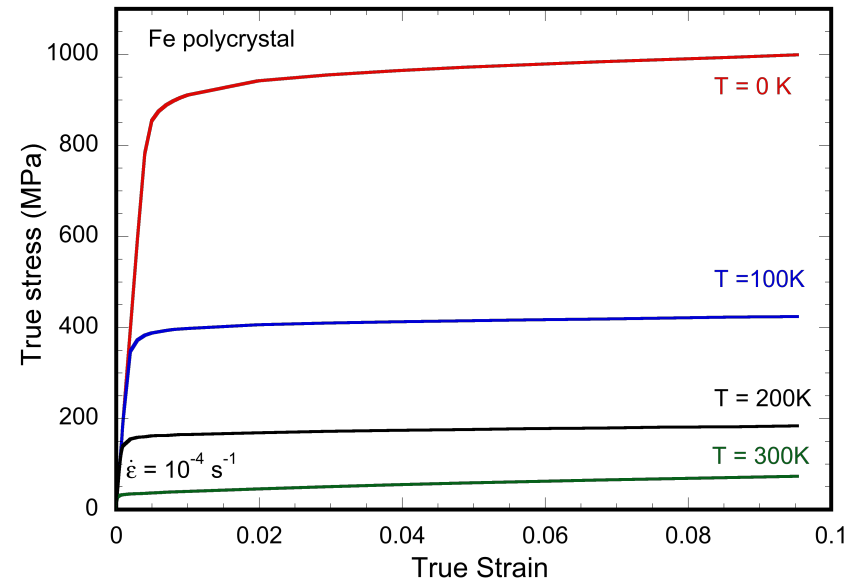

(b) Predicted stress-strain responses

Figure 15: (a) Finite element model of Fe polycrystal using 125,000 hexahedral elements and (b) predicted stressstrain responses at different temperatures.



(a)

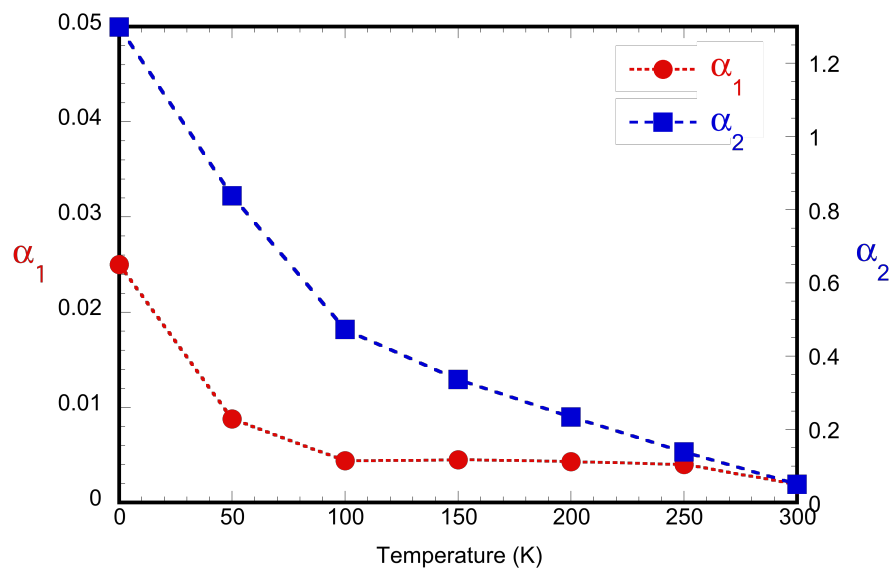

(b)

Figure 16: Plots of (a) predicted (data points) and fitted (solid lines) polycrystal biaxial yield surfaces at $0 \mathrm{~K}, 100 \mathrm{~K}$ and $300 \mathrm{~K}$ and (b) best-fit $\alpha_{1}$ and $\alpha_{2}$ at different temperatures. 
Using the least-squares fit to polycrystalline CP-FE results, the best-fit values of $\alpha_{1}$ and $\alpha_{2}$ at different temperatures are obtained. For the fitted yield surfaces to be formally convex, $\alpha_{2} \leq 3 \sqrt{3} / 4$, and is enforced in all of the fitting procedures. Solid lines in Figure 16 (a) shows the fitted yield surface using Equation (20). This demonstrates that the polycrystalline yield criteria in Equation (20) accurately reproduces simulated yield surface of polycrystalline Fe. As shown in Figure 16 (b), the best-fit $\alpha_{1}$ and $\alpha_{2}$ decrease with increasing temperature and converge to isotropic von Mises yield function at high temperatures $\left(\alpha_{1}=\alpha_{2}=0\right)$.

\section{Conclusions}

The flow behavior in BCC metals, such as Fe, at low temperatures deviates from that of standard FCC metals. The current work focuses on considering these attributes to accurately simulate the deformation behavior of Fe. Atomistic simulations are used to quantify the non-Schmid effects and formulate a temperature and strain rate dependent enthalpy model. A novel activation-enthalpy based model parameterized by atomistic simulations and single crystal experiments is implemented into crystal plasticity framework to simulate plastic deformations of single and polycrystalline Fe. Comparisons of model predictions with experimental data clearly demonstrate the importance of correctly capturing the temperature and strain rate dependent non-Schmid effects.

Although the proposed CP-FE model captures some aspects of deformation behaviors in Fe more accurately than prior Schmid law based models, there are several aspects that would require further investigation. There is still an ambiguity of operating slip planes in Fe, e.g. $\{110\},\{112\}$ and $\{123\}$ slips are observed from slip trace analysis (Spitzig and Keh, 1970) which may have modest effects on the results presented here. The current CP-FE model can be further improved by incorporating non-local effects and more sophisticated treatment at grain boundaries and their interactions with dislocation. Despite many limitations, the framework developed in this work conveniently bridges different length scales and provides a basis for a practical multi-scale model that can more accurately capture the fundamental mechanisms of plasticity.

The key findings from this work are as follows.

- A novel flow rule based on the generalized non-Schmid law and the line tension model is proposed and parameterized with atomistic simulations and single crystal experiments. The 
model is implemented into a BCC finite element crystal plasticity model.

- Atomistically-informed CP-FE model for Fe more accurately predicts temperature and strain rate effects as well as crystal orientation dependent yield stresses of single crystals found in the literature as compared to a Schmid-based model.

- The model shows that the Non-Schmid stresses affect stress-strain responses as well as texture evolution. Non-Schmid effects are more significant at low temperatures and high strain rates.

- CP-FE simulations of iron polycrystals demonstrated that the biaxial yield surface deviate from isotropic von-Mises yield surface, especially at low temperature, and converges to isotropic yield surface as temperature is increased. Effects of both non-Schmid stresses and pressure dependence decreased with increasing temperature.

\section{Acknowledgements}

Sandia National Laboratories is a multi-program laboratory managed and operated by Sandia Corporation, a wholly owned subsidiary of Lockheed Martin Corporation, for the U.S. Department of Energy's National Nuclear Security Administration under contract DE-AC04-94AL85000. 


\section{Appendix: Applied Stress Tensor and the Associated CRSS Criterion}

In section 3.3, four stress tensors were applied to obtain non-Schmid constants and $\tau_{c r}$. Stress tensors in Table 1 are applied with respect to MRSSP (xyz-coordinate system) as shown in in Figure 3 (a).

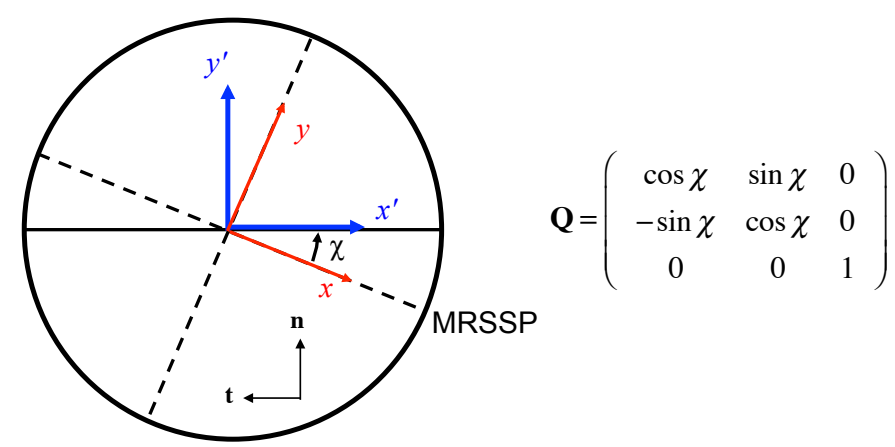

Figure 17: Schematics showing the orientation of the applied stress. The stress tensor is applied to $x y z$-coordinate system.

To find directions of stress tensors in $\mathbf{m}, \mathbf{n}$ and $\mathbf{t}$ directions, $x y z$-coordinate system is transformed to $x^{\prime} y^{\prime} z^{\prime}$-system using $\mathbf{Q}$ matrix as shown in Figure 17 . As shown in Figure 17, $x y z-$ coordinate system is rotated counterclockwise by angle $\chi$ about the $z$ axis. The stress tensor on the rotated coordinate system, $\boldsymbol{\sigma}^{\prime}$, can be represented as follows:

$$
\boldsymbol{\sigma}^{\prime}=\mathbf{Q} \cdot \boldsymbol{\sigma} \cdot \mathbf{Q}^{T}
$$

Equations (22)-(25) show the stress tensors in Table 1 transformed to the $x^{\prime} y^{\prime} z^{\prime}$-system.

$$
\begin{aligned}
\boldsymbol{\sigma}_{\tau^{\mathrm{MRSSP}}}^{\prime} & =\left[\begin{array}{ccc}
0 & 0 & \tau^{\mathrm{MRSSP}} \sin \chi \\
0 & 0 & \tau^{\mathrm{MRSSP}} \cos \chi \\
\tau^{\mathrm{MRSSP}} \sin \chi & \tau^{\mathrm{MRSSP}} \cos \chi & 0
\end{array}\right] \\
\boldsymbol{\sigma}_{\tau_{\mathrm{MRSSP}}^{\prime}, \tau_{1}^{\perp}}^{\prime} & {\left[\begin{array}{ccc}
-\tau_{1}^{\perp} \cos 2 \chi & \tau_{1}^{\perp} \sin 2 \chi & \tau^{\mathrm{MRSSP}} \sin \chi \\
\tau_{1}^{\perp} \sin 2 \chi & \tau_{1}^{\perp} \cos 2 \chi & \tau^{\mathrm{MRSSP}} \cos \chi \\
\tau^{\mathrm{MRSSP}} \sin \chi & \tau^{\mathrm{MRSSP}} \cos \chi & 0
\end{array}\right] }
\end{aligned}
$$




$$
\begin{aligned}
\boldsymbol{\sigma}_{\tau^{\mathrm{MRSSP}}, \tau_{2}^{\perp}}^{\prime} & =\left[\begin{array}{ccc}
\tau_{2}^{\perp} \sin ^{2} \chi & \tau_{2}^{\perp} \sin \chi \cos \chi & \tau^{\mathrm{MRSSP}} \sin \chi \\
\tau_{2}^{\perp} \sin \chi \cos \chi & \tau_{2}^{\perp} \cos ^{2} \chi & \tau^{\mathrm{MRSSP}} \cos \chi \\
\tau^{\mathrm{MRSSP}} \sin \chi & \tau^{\mathrm{MRSSP}} \cos \chi & -\tau_{2}^{\perp}
\end{array}\right] \\
\boldsymbol{\sigma}_{\tau^{\mathrm{MRSSP}}, P}^{\prime} & =\left[\begin{array}{ccc}
P & 0 & \tau^{\mathrm{MRSSP}} \sin \chi \\
0 & P & \tau^{\mathrm{MRSSP}} \cos \chi \\
\tau^{\mathrm{MRSSP}} \sin \chi & \tau^{\mathrm{MRSSP}} \cos \chi & P
\end{array}\right]
\end{aligned}
$$

Through the $\chi$ dependency, Equation (22) shows that $\tau^{\text {MRSSP }}$ has components that act both on the Schmid slip system $\left(\sigma_{y^{\prime} z^{\prime}}\right)$ and normal to it but still parallel to the dislocation line $\left(\sigma_{x^{\prime} z^{\prime}}\right)$. The remaining stress components are then explored through the addition of the independent shear stresses $\tau_{1}^{\perp}$ and $\tau_{2}^{\perp}$ applied perpendicular to the slip direction. Finally, as mentioned in the main article, the pressure dependency is included to decouple the tensile components.

The Schmid tensor and non-Schmid stress projection tensors along $x^{\prime} y^{\prime} z^{\prime}$-coordinate system can be represented as follows:

$$
\begin{array}{r}
\mathbf{P}_{S}=\left[\begin{array}{lll}
0 & 0 & 0 \\
0 & 0 & \frac{1}{2} \\
0 & \frac{1}{2} & 0
\end{array}\right] \\
\mathbf{P}_{n S}=c_{1}\left[\begin{array}{ccc}
0 & 0 & -\frac{1}{2} \\
0 & 0 & 0 \\
-\frac{1}{2} & 0 & 0
\end{array}\right]+c_{2}\left[\begin{array}{ccc}
0 & -\frac{1}{2} & 0 \\
-\frac{1}{2} & 0 & 0 \\
0 & 0 & 0
\end{array}\right]+c_{3}\left[\begin{array}{lll}
0 & 0 & 0 \\
0 & 1 & 0 \\
0 & 0 & 0
\end{array}\right]+c_{4}\left[\begin{array}{lll}
1 & 0 & 0 \\
0 & 0 & 0 \\
0 & 0 & 0
\end{array}\right]+c_{5}\left[\begin{array}{lll}
0 & 0 & 0 \\
0 & 0 & 0 \\
0 & 0 & 1
\end{array}\right]=\left[\begin{array}{ccc}
c_{4} & -\frac{c_{2}}{2} & -\frac{c_{1}}{2} \\
-\frac{c_{2}}{2} & c_{3} & 0 \\
-\frac{c_{1}}{2} & 0 & c_{5}
\end{array}\right] .
\end{array}
$$

Using Equations (3), (26), (27), the following relation is satisfied:

$$
\tau_{c r}=\mathbf{P}_{S}: \boldsymbol{\sigma}+\mathbf{P}_{n S}: \boldsymbol{\sigma}=\left[\begin{array}{ccc}
c_{4} & -\frac{c_{2}}{2} & -\frac{c_{1}}{2} \\
-\frac{c_{2}}{2} & c_{3} & \frac{1}{2} \\
-\frac{c_{1}}{2} & \frac{1}{2} & c_{5}
\end{array}\right]: \boldsymbol{\sigma}^{\prime}
$$

The CRSS criterion for each stress tensor can be derived by using Equation (28) and substituting $\tau^{\mathrm{MRSSP}}$ with CRSS. For example, by using Equations (24) and (28), the following relationship 
between $\tau^{\mathrm{MRSSP}}, \tau_{c r}, \chi$ and $c_{i}$ can be derived as follows:

$$
\tau_{c r}=\tau^{\operatorname{MRSSP}}\left(\cos \chi-c_{1} \sin \chi\right)+\tau_{2}^{\perp}\left(-c_{2} \sin \chi \cos \chi+\left(c_{3}-c_{4}\right) \cos ^{2} \chi+\left(c_{4}-c_{5}\right)\right) .
$$

By substituting $\tau^{\mathrm{MRSSP}}$ with $C R S S$, the following relation can be derived for $C R S S\left(\chi, \tau_{2}^{\perp}\right)$ :

$$
C R S S\left(\chi, \tau_{2}^{\perp}\right)=\frac{\tau_{c r}-\tau_{2}^{\perp}\left(-c_{2} \sin \chi \cos \chi+\left(c_{3}-c_{4}\right) \cos ^{2} \chi+\left(c_{4}-c_{5}\right)\right)}{\cos \chi-c_{1} \sin \chi}
$$

Similarly, $C R S S(\chi), C R S S\left(\chi, \tau_{1}^{\perp}\right)$ and $C R S S(\chi, P)$ in Table 1 can be derived by applying $\boldsymbol{\sigma}_{\tau^{\mathrm{MRSSP}}}^{\prime}$, $\boldsymbol{\sigma}_{\tau^{\mathrm{MRSSP}}, \tau_{1}^{\perp}}^{\prime}$ and $\boldsymbol{\sigma}_{\tau_{\mathrm{MRSSP}}^{\prime}, P}^{\prime}$ in Equations (22), (23) and (25), respectively. 


\section{References}

Argon, A., 2008. Strengthening mechanisms in crystal plasticity. Oxford University Press.

Asaro, R. J., 1983. Micromechanics of crystals and polycrystals. Adv. Appl. Mech. 23, 1-115.

Barvinschi, B., Proville, L., Rodney, D., 2014. Quantum peierls stress of straight and kinked dislocations and effect of non-glide stresses. Modelling and Simulation in Materials Science and Engineering 22, 25006-25019.

Becker, C. A., Tavazzaa, F., Trautta, Z. T., de Macedoc, R. A. B., 2013. Considerations for choosing and using force fields and interatomic potentials in materials science and engineering. Current Opinion in Solid State and Materials Science 17, 277-283.

Biffle, J. H., 1987. A three-dimensional finite element computer program for the nonlinear quasistatic response of solids with the conjugate gradient method, SAND87-1305. Sandia National Laboratories.

Bronkhorst, C. A., Kalidindi, S. R., Anand, L., 1992. Polycrystalline plasticity and the evolution of crystallographic texture in fcc metals. Philos. Trans. Roy. Soc. London A 341, 443.

Brunner, D., 2000. Comparison of flow-stress measurements on high-purity tungsten single crystals with the kink-pair theory. Mater. Trans. JIM 41, 152-160.

Brunner, D., Diehl, J., 1991a. Strain-rate and temperature dependence of the tensile flow stress of high-purity $\alpha$-iron above $250 \mathrm{k}$ (regime i) studied by means of stress-relaxation tests. Phys. Status Solidi A 124, 155-170.

Brunner, D., Diehl, J., 1991b. Temperature and strain-rate dependence of the tensile flow stress of high-purity $\alpha$-iron below $250 \mathrm{k}$ i. stress/temperature regime iii. Phys. Status Solidi A 124, $355-464$.

Buchheit, T. E., Wellman, G. W., Battaile, C. C., 2005. Investigating the limits of polycrystal plasticity modeling. Int. J. Plasticity 21, 221-249.

Butt, M. Z., 2007. Kinetics of flow stress in crystals with high intrinsic lattice friction. Philos. Mag. $87,3595-3614$. 
Cazacu, O., Barlat, F., 2004. A criterion for description of anisotropy and yield differential effects in pressure-insensitive metals. International Journal of Plasticity 20, 2027-2045.

Chamati, H., Papanicolaou, N. O., Mishin, Y., Papaconstantopoulos, D. A., 2006. Embedded-atom potential for fe and its application to self-diffusion on fe(100). Surf. Sci. 600, 1793-1803.

Christian, J. W., 1983. Some surprising features of the plastic deformation of body-centered-cubic metals and alloys. Met. Trans. A 14A, 1237-1256.

Dao, M., Asaro, R. J., 1993. Non-schmid effects and localized plastic flow in intermetallic alloys. Mat. Sci. Eng. A A179, 143-160.

Drucker, D. C., 1949. Relations of experiments to mathematical theories of plasticity. Journal of Applied Mechanics 16, 349-357.

Drucker, D. C., Prager, W., 1952. Soil mechanics and plastic analysis for limit design. Quarterly of Applied Mathematics 10, 157-165.

Duesbery, M. S., Vitek, V., 1998. Plastic anisotropy in B.C.C. transition metals. Acta. Mater. 46, $1481-1492$.

Duesbery, M. S., Vitek, V., Bowen, D. K., 1973. Effect of shear-stress on screw dislocation core structure in body-centered cubic lattices. Proc. Roy. Soc. Lond. A Mat. 332, 85-111.

Fellinger, M. R., Park, H., Wilkins, J. W., 2010. Force-matched embedded-atom method potential for niobium. Phys. Rev. B 81, 144119.

Gordon, P., Neeraj, T., Mendelev, M., 2011. Screw dislocation mobility in bcc metals: a refined potential description for $\alpha$-fe. Philos. Mag. 91, 393-3945.

Gordon, P. A., Neeraj, T., Li, Y., Li, J., 2010. Screw dislocation mobility in bcc metals: the role of the compact core on double-kink nucleation. Model. Simul. Mater. Sci. Eng. 18, 085008.

Groeber, M., Ghosh, S., Uchic, M., Dimiduk, D., 2008a. A framework for automated analysis and simulation of 3d polycrystalline microstructures. part 1: Statistical characterization. Acta Mater. $56,1257-1273$. 
Groeber, M., Ghosh, S., Uchic, M., Dimiduk, D., 2008b. A framework for automated analysis and simulation of $3 \mathrm{~d}$ polycrystalline microstructures. part 2: Synthetic structure generation. Acta Mater. 56, 1274-1287.

Gröger, R., Bailey, A., Vitek, V., 2008a. Multiscale modeling of plastic deformation of molybedenum and tungsten: I. Atomistic studies of the core structure and glide of $1 / 2\langle 111\rangle$ screw dislocations at 0 K. Acta. Mater. 56, 5401-5411.

Gröger, R., Racherla, V., Basani, J., Vitek, V., 2008b. Multiscale modeling of plastic deformation of molybedenum and tungsten: II. Yield criterion for single crystals based on atomsitcs studies of glide of $1 / 2\langle 111\rangle$ screw dislocations. Acta. Mater. 56, 5412-5425.

Gröger, R., Racherla, V., Basani, J., Vitek, V., 2008c. Multiscale modeling of plastic deformation of molybedenum and tungsten: III. Effects of temperature and plastic strain rate. Acta. Mater. $56,5426-5439$.

Hale, L. M., Lim, H., Zimmerman, J. A., Battaile, C. C., Weinberger, C. R., 2014a. Insights on activation enthalpy for non-schmid slip in bcc metals. Scripta Mater. Accepted for publication.

Hale, L. M., Zimmerman, J. A., Weinberger, C. R., 2014b. Simulations of bcc tantalum screw dislocations: Why classical inter-atomic potentials predict $\{112\}$ slip. Computational Materials Science 90, 106-115.

Henkelman, G., Jonsson, H., 2000. Improved tangent estimate in the nudged elastic band method for finding minimum energy paths and saddle points. J. Chem. Phys. 113, 9978-9985.

Henkelman, G., Uberuaga, B. P., Jonsson, H., 2000. Ia climbing image nudged elastic band method for finding saddle points and minimum energy paths. J. Chem. Phys. 113, 9901-9904.

Hirsch, P. B., 1960. Proceedings of the fifth international conference on crystallography. Cambridge University Press, p. 139.

Hirth, J., Lothe, J., 1982. Theory of Dislocations. Krieger.

Hollang, L., Brunner, D., Seeger, A., 2001. Work hardening and flow stress of ultrapure molybdenum single crystals. Mater. Sci. Eng. A 319, 233-236. 
Hollang, L., Hommel, M., Seeger, A., 1997. The flow stress of ultra-high-purity molybdenum single crystals. Phys. Status Solidi A 160, 329-354.

Hutchinson, J. W., 1976. Bounds and self-consistent estimates for creep of polycrystalline materials. Proc. R. Soc. Lond. A 348, 101-127.

Ito, K., Vitek, V., 2001. Atomistic study of non-Schmid effects in the plastic yielding of bcc metals. Philos. Mag. A 81, 1387-1407.

Kalidindi, S. R., Bronkhorst, C. A., Anand, L., 1992. Crystallographic texture evolution in bulk deformation processing of fcc metals. J. Mech. Phys. Solids 40, 537.

Kocks, U. F., 1976. Laws for work-hardening and low-temperature creep. J. Eng. Mater. Tech., ASME 98, 76-85.

Kocks, U. F., Argon, A. S., Ashby, M. F., 1975. Thermodynamics and kinetics of slip. Progress in Materials Science 19, 1-289.

Koester, A., Ma, A., Hartmaier, A., 2012. Atomistically informed crystal plasticity model for bodycentered cubic iron. Acta Mater. 60, 3894-3901.

Kuramoto, E., Aono, Y., Kitajima, K., 1979a. Thermally activated slip deformation of high-purity iron single-crystals between 4.2-k and 300-k. Scripta Metallurgica 13, 1039-1042.

Kuramoto, E., Aono, Y., Kitajima, K., Makaeda, K., Takeuchi, S., 1979b. Thermally activated slip deformation between 0.7-k and 77-k in high-purity iron single-crystals. Phil. Mag. A 39, 717-724.

Lee, E. H., 1969. Elastic-plastic deformation at finite strains. Appl. Mech. 36, 1-6.

Lee, M. G., Lim, H., Kim, J. H., Adams, B. L., Wagoner, R. H., 2010. A dislocation density-based single crystal constitutive equation. Int. J. Plasticity 26, 925-938.

Lim, H., Battaile, C. C., Carroll, J. D., Boyce, B. L., Weinberger, C. R., 2015. A physically based temperature and strain rate dependent crystal plasticity model for bcc metals. J. Mech. Phys. Sol. $74,80-96$. 
Lim, H., Carroll, J. D., Battaile, C. C., Buchheit, T. E., Boyce, B. L., Weinberger, C. R., 2014. Grain-scale experimental validation of crystal plasticity finite element simulations of tantalum oligocrystals. Int. J. Plasticity 60, 1-18.

Lim, H., Weinberger, C. R., Battaile, C. C., Buchheit, T. E., 2013. Application of generalized nonSchmid yield law to low temperature plasticity in bcc transition metals. Model. Simul. Mater. Sci. Eng. 21, 045015.

Peirce, D., Asaro, R. J., Needleman, A., 1982. An analysis of nonuniform and localized deformation in ductile single crystals. Acta Metall. 30, 1087-1119.

Plimpton, S., 1995. Fast parallel algorithms for short-range molecular-dynamics. Journal of Computational Physics 117, 1-19.

Proville, L., Ventelon, L., Rodney, D., 2013. Prediction of the kink-pair formation enthalpy on screw dislocations in alpha-iron by a line tension model parametrized on empirical potentials and first-principles calculations. Phys. Rev. B 87, 144106.

Qin, Q., Bassani, J. L., 1992a. Non-associated plastic flow in single crystals. J. Mech. Phys. Solids $40,835-862$.

Qin, Q., Bassani, J. L., 1992b. Non-Schmid yield behavior in single crystals. J. Mech. Phys. Solids $40,813-833$.

Quesnel, D. J., Sato, A., Meshii, M., 1975. Solution softening and hardening in the iron-carbon system. Mater. Sci. Eng. 18, 199-208.

Rao, S., Woodward, C., 2001. Atomistic simulations of $(\mathrm{a} / 2)<111>$ screw dislocations in bcc mo using a model generalized pseudo-potential theory potential. Philos. Mag. A 81, 1317-1327.

Rodney, D., 2007. Activation enthalpy for kink-pair nucleation on dislocations: Comparison between static and dynamic atomic-scale simulations. Phys. Rev. B 76, 144108.

Saylor, D., Fridy, J., El-Dasher, B., Jung, K.-Y., Rollett, A., 2004. Statistically representative three-dimensional microstructures based on orthogonal observation sections. Meta. Trans. A 35, 1969-1979. 
Seeger, A., 1981. The temperature and strain-rate dependence of the flow stress of body-centered cubic metals: A theory based on kink-kink interactions. Z. Metallikd 72, 369-380.

Seeger, A., 2001. Why anomalous slip in body-centred cubic metals? Mater. Sci. Eng. A 319-321, $254-260$.

Seeger, A., Holzwarth, U., 2006. Slip planes and kink properties of screw dislocations in high-purity niobium. Philos. Mag. 86, 3861-3892.

Spitzig, W. A., Keh, A. S., 1970. Orientation and temperature dependence of slip in iron single crystals. Met. Trans. B 1, 2751-2757.

Srivastava, K., Gröger, R., Weygand, D., Gumbsch, P., 2013. Dislocation motion in tungsten: Atomistic input to discrete dislocation simulations. Int. J. Plasticity 47, 126-142.

Steinmann, P., Kuhl, E., Stein, E., 1998. Aspects of non-associated single crystal plasticity: Influence of non-schmid effects and localization analysis. Int. J. Sol. Struc. 35, 4437-4456.

Stroh, A. N., 1958. Dislocations and cracks in anisotropic elasticity. Phil. Mag. 3, 625-646.

Stroh, A. N., 1962. Steady state problems in anisotropic elasticity. J. Math. Phys. Camb. 41, 77-103.

Takeuchi, S., 1979. Core structure of a screw dislocation in the b.c.c. lattice and its relation to slip behavior of $\alpha$-iron. Philos. Mag. A 39, 661-671.

Takeuchi, S., Kuramoto, E., 1975. Thermaly activated motion of a screw dislocation in a model b.c.c. crystal. J. Phys. Soc. Jpn. 38, 480-487.

Taylor, G. I., 1934. The mechanism of plastic deformation of crystals. Part I. Theoretical. Proc. Roy. Soc. A 165, 362-387.

Ventelon, L., Willaime, F., 2007. Core structure and peierls potential of screw dislocations in alpha-fe from first principles: cluster versus dipole approaches. J. Comput.-Aided Mater. Des. $14,85-94$.

Ventelon, L., Willaime, F., 2010. Generalized stacking-faults and screw-dislocation core-structure in bcc iron: A comparison between ab initio calculations and empirical potentials (vol 90, pg 1063, 2010). Philos. Mag. 90, 4071-4071. 
Wang, G. F., Strachan, A., Cagin, T., Goddard, W. A., 2002. Kinks in the a/2 〈111〉 screw dislocation in ta. J. Comput-Aided Mater. 8, 117-125.

Wang, Z., Beyerlein, I., 2010. An atomistically-informed dislocation dynamics model for the plastic anisotropy tension and compression asymmetry of bcc metals. Int. J. Plasticity.

Weinberger, C. R., Battaile, C. C., Buchheit, T. E., Holm, E. A., 2012. Incorporating atomistic models of lattice friction into bcc crystal plasticity models. Int. J. Plasticity 37, 16-30.

Weinberger, C. R., Boyce, B. L., Battaile, C. C., 2013a. Slip planes in bcc transition metals. Int. Mater. Rev. 58, 296-314.

Weinberger, C. R., Tucker, G. J., Foiles, S., 2013b. The peierls potential of screw dislocations in bcc transition metals: predictions form density functional theory. Phys. Rev. B 87, 054114.

Werner, M., 1987. Temperature and strain-rate dependence of the flow stress of ultrapure tantalum single crystal. Phys. Status Solidi A 104, 63-78.

Yalcinkaya, T., Brekelmans, W. A. M., Geers, M. G. D., 2008. Bcc single crystal plasticity modeling and its experimental identification. Modell. Simul. Mater. Sci. Eng. 16, 085007. 